\title{
Equivalence of QCD in the $\epsilon$-regime and chiral Random Matrix Theory with or without chemical potential
}

\author{
F. BAsile ${ }^{1,2}$ and G. Akemann ${ }^{1,3}$ \\ ${ }^{1}$ Department of Mathematical Sciences \& BURSt Research Centre \\ Brunel University West London \\ Uxbridge UB8 3PH, United Kingdom \\ ${ }^{2}$ Dipartimento di Fisica dell'Università di Pisa \& INFN \\ Largo B. Contecorvo 3 Ed. C, 56127 Pisa, Italy \\ ${ }^{3}$ Centre de Physique Théorique, CNRS Luminy, \\ Case 907, F-13288 Marseille Cedex 9, France*
}

October 28, 2018

\begin{abstract}
We prove that QCD in the $\epsilon$-regime of chiral Perturbation Theory is equivalent to chiral Random Matrix Theory for zero and both non-zero real and imaginary chemical potential $\mu$. To this aim we prove a theorem that relates integrals over fermionic and bosonic variables to super-Hermitian or super-Unitary groups also called superbosonisation. Our findings extend previous results for the equivalence of the partition functions, spectral densities and the quenched two-point densities. We can show that all $k$-point density correlation functions agree in both theories for an arbitrary number of quark flavours, for either $\mu=0$ or $\mu \neq 0$ taking real or imaginary values. This implies the equivalence for all individual $k$-th eigenvalue distributions which are particularly useful to determine low energy constants from Lattice QCD with chiral fermions.
\end{abstract}

CPT-P33-2007

${ }^{*}$ CPT is "UMR 6207 du CNRS et des universités d'Aix-Marseille I, d'Aix-Marseille II et du Sud Toulon-Var, affiliée à la FRUMAM" 


\section{Introduction}

The application of chiral Random Matrix Theory (chRMT) to QCD, initially introduced at zero chemical potential in [1, has led to many exact analytical results for the Dirac operator spectrum. These have in turn found applications in comparison to Lattice Gauge theory by various groups (for reviews see [2, 3]). The deeper reason for this analytical understanding of the Dirac spectrum in the phase with broken chiral symmetry can be traced back to the relation between chRMT and the epsilon regime of chiral Perturbation Theory $(\epsilon \chi \mathrm{PT})$. Being based on global symmetries alone there are three classes of chiral symmetry breaking patterns, corresponding to the three possible anti-unitary symmetries that lead to the unitary, orthogonal and symplectic chRMT [4]. Here, we will only report on the first class containing QCD, which is technically the simplest. Relatively little is known about the equivalence to $\epsilon \chi \mathrm{PT}$ for gauge theories in the two other classes, see however [5, 6].

In this paper we will show that any correlation function of Dirac operator eigenvalues computed starting from $\epsilon \chi \mathrm{PT}$ is the same as the corresponding quantity computed from chRMT in the large- $N$ limit of infinite matrices, corresponding to the infinite volume limit of $\chi \mathrm{PT}$. This equivalence holds both with and without chemical potential, being of real or imaginary type. The chRMT we use is the one introduced by Shuryak and Verbaarschot [1] for $\mu=0$, by Osborn [7] for real $\mu \neq 0$, and by Akemann et al. [8] for imaginary $\mu$ where the two latter are two-matrix models. The one-matrix model for real $\mu \neq 0$ introduced earlier by Stephanov [9] can also be mapped to $\epsilon \chi \mathrm{PT}$ and is thus equivalent to [7. Being technically more involved we don't give details of our analogous equivalence proof here.

The $\epsilon \chi \mathrm{PT}$ - chRMT equivalence is established by relating the generating functionals of resolvents of an arbitrary finite degree, with any number of flavours $N_{f}$. In $\epsilon \chi \mathrm{PT}$ these generating functionals are obtained by adding additional fermionic and bosonic quarks, leading to supergroup integrals. For real $\mu \neq 0$ it is nontrivial to make them convergent, and our result for the corresponding $\epsilon \chi \mathrm{PT}$ is new. These group integrals are then shown to be equal to the corresponding quantities in chRMT at large- $N$, given by the expectation value of ratios of Dirac operator determinants (also called characteristic polynomials in the chRMT language).

The technical tool we use is a theorem we prove also called superbosonisation, which was obtained independently by [10, 11]. After writing the ratios of determinants in terms of superfermionic variables and integrating out the Gaussian random matrices the theorem allows us to map these expressions to the supergroup integrals of $\epsilon \chi \mathrm{PT}$. We only take the large- $N$ limit at the very end, without performing any saddle point approximations. Let us emphasize that although there are many applications of RMT in Physics [12, only in few cases an exact map to the underlying microscopic theory has been achieved.

In chRMT there are alternative ways to compute arbitrary $k$-point density correlations, or individual eigenvalue correlation functions, some without introducing resolvents. One example is the method of orthogonal polynomials [13. On the other hand the supergroup integrals in $\epsilon \chi \mathrm{PT}$ are increasingly hard to calculate for $k>1$. By establishing an equivalence for all generating functionals we can therefore match with all known chRMT correlation functions, were they obtained using resolvents or not. For $\mu=0$ all generating functionals in chRMT were computed in [14, 15. For real $\mu \neq 0$ all building blocks for characteristic polynomials in chRMT in the large- $N$ limit follow from [16] and [17. At imaginary $\mu \neq 0$ so far only the bi-orthogonal polynomial method has been used in chRMT 8 .

The explicit results for the $k$-point density correlation functions can be most conveniently read off directly from [18 for massless, and from [19, 20] for massive correlations at $\mu=0$, for both massive and massless correlations from [16] at real $\mu \neq 0$, and likewise from 8 for imaginary $\mu$. The corresponding individual eigenvalue correlation functions that require the knowledge of all density correlations were obtained in [21] for $\mu=0$, in 22 for real $\mu$ and in 23 for imaginary $\mu$.

The following results were previously known about the $\epsilon \chi \mathrm{PT}-$ chRMT equivalence: partition functions with only fermions were shown to agree for zero [1] and non-zero $\mu$ [24, 25, 26, 3]. The equivalence for partition functions with only bosons at $\mu=0$ follows from [27. The generating functional of a $\left(N_{f}+1 \mid 1\right)$ supergroup integral leading to the spectral density was computed in [28, 29] including $N_{f}$ massless fermions. This work was extended to include the quenched two-point density in [30, in this case too only for $\mu=0$. Furthermore, the quenched density at real $\mu \neq 0$ was computed from both $\epsilon \chi \mathrm{PT}[14$ ] and chRMT [7] and found to be in agreement. For imaginary isospin $\mu \neq 0$ the equivalence was established up to the two-point function in [31, 8]. It was pointed out 32 3 how in principle to compute the distributions of the $k$-th individual eigenvalue from $\epsilon \chi \mathrm{PT}$, using all $k$-point density correlation functions, in order to reproduce previous chRMT results 21. The same strategy can be applied for non-zero $\mu[23,22]$. 
Our equivalence proof for all $k$-point density correlation functions thus, in particular, fills this gap. Individual eigenvalue distributions have been used in several groups to compare with Lattice results with exact or approximate chiral symmetry, leading to a well defined gauge field topology.

A further remark concerns universality. On the chRMT side it is well known for $\mu=0$ that in the microscopic large- $N$ limit the results hold for a much wider class than Gaussian weight functions [18, 33, 19. For that reason we can restrict ourselves to establish the equivalence for Gaussian chRMT. For $\mu \neq 0$ less is known. Both the model by Stephanov 9] and by Osborn [7] are Gaussian matrix models. As already mentioned above the fermionic partition functions [24, 25, 26, 3] and the quenched density [14, 7] were shown to agree among both matrix models, and with $\epsilon \chi \mathrm{PT}$. We can now extend this equivalence to all correlation functions. This agreement suggests universality for chRMT in the broken phase to hold at $\mu \neq 0$ as well.

Finally let us mention that for non-chiral RMT the equivalence to an effective theory for spontaneous flavour symmetry breaking in 3D is simpler and has already been fully established at $\mu=0$ by Szabo 34. We expect that an extension to $\mu \neq 0$ can be done following the same lines as here.

Our paper is organised as follows: In section 2 the resolvent method is briefly recalled both for Hermitian operators $(\mu=0, \mu$ pure imaginary) and non-Hermitian ones ( $\mu$ real), in order to clarify how the $k$-point correlation functions are obtained from a partially quenched (pq) theory. In sections 3 , 4 and 5 the proofs of the equivalence for the pq theories for $\mu=0$, for imaginary $\mu$ and real $\mu$ are given, respectively. Here we write the averages over ratios of Dirac determinants in chRMT as supervectors integrals, perform the chRMT average and apply our theorem relating to $\epsilon \chi \mathrm{PT}$ supergroup integrals. In particular the form of pq $\epsilon \chi \mathrm{PT}$ with real $\mu \neq 0$ is given. The superbosonisation theorem is then presented and proved in section 6. More technical remarks on delta functions and on some integrals used are deferred to appendices $\mathrm{A}$ and $\mathrm{B}$

\section{The resolvent method}

The resolvent method is a way to compute the $k$-point eigenvalue correlation function of an operator distributed according to a given ensemble (in our case these ensembles are QCD or chRMT). Starting from the expectation value of a ratio of characteristic polynomials, this quantity may be considered as the partition function of a theory with an additional number of fermionic and bosonic quarks, called partially quenched QCD (or chRMT). Both supersymmetric and replica methods rely on the resolvent method.

In this section we briefly summarise it's idea for the reader's convenience, referring to the literature for more detailed explanations [35. Let us emphasize that the resolvent method is substantially different whether one considers a theory with an Hermitian or non-Hermitian operator. QCD with zero or imaginary chemical potential is a theory with an anti-Hermitian Dirac operator, the correspondence with an Hermitian operator is trivial. QCD with real chemical potential is a non-Hermitian theory.

We start by showing the resolvent method for Hermitian theories. The $k$-point correlation function is defined as the expectation value of the product of $k \delta$-functions:

$$
\rho_{k}\left(\eta_{1}, \ldots, \eta_{k}\right) \equiv\left\langle\prod_{j=1}^{k} \sum_{\lambda \in \mathrm{e} . \mathrm{v} .} \delta\left(\eta_{j}-\lambda\right)\right\rangle
$$

where the expectation value is computed according to the ensemble of the operator $D$ whose eigenvalues are $\lambda$. Alternative definitions of this quantity may be given, in order to avoid the contact terms obtained through delta functions at coinciding arguments $\eta_{i}=\eta_{j}[12$.

For simplicity we consider first the easiest case, the spectral density $(k=1)$. We define its resolvent as

$$
G_{1}(z) \equiv\left\langle\sum_{\lambda \in \mathrm{e} . \mathrm{v} .} \frac{1}{z-\lambda}\right\rangle=\int d \lambda \rho_{1}(\lambda) \frac{1}{z-\lambda} .
$$

Here the argument $z$ is taken to lie outside the support of $D \subset \mathcal{R}$. Given that the density is $z$-independent this integral equation can be inverted as follows [36:

$$
\rho_{1}(\eta)=\frac{1}{2 \pi i} \lim _{\varepsilon \rightarrow 0^{+}}\left[G_{1}(\eta-i \varepsilon)-G_{1}(\eta+i \varepsilon)\right]
$$


taking the discontinuity of the resolvent when crossing the support. An essential ingredient for this result is the fact that all the eigenvalues of an (anti-)Hermitian operator lie on the real (imaginary) axis. This requirement is not fulfilled by theories with a non-Hermitian Dirac operator, invalidating these equations. The next step is to generate the resolvent (2) as a ratio of determinants:

$$
G_{1}(z)=\left\langle\operatorname{Tr}\left[\frac{1}{z-D}\right]\right\rangle=\left.\frac{\partial}{\partial z^{\prime}}\left\langle\frac{\operatorname{Det}\left[D-z^{\prime}\right]}{\operatorname{Det}[D-z]}\right\rangle\right|_{z^{\prime}=z} .
$$

The generalisation to the $k$-point correlation function is conceptually not different, with a bit more attention

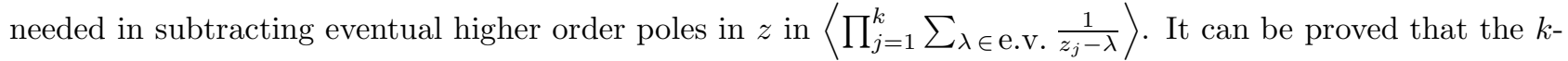
point correlation function may be generated through the theory with $k$ additional bosonic and fermionic quarks. The corresponding resolvents are defined as

$$
G_{k}\left(z_{1}, \ldots, z_{k}\right) \equiv\left\langle\prod_{j=1}^{k} \sum_{\lambda \in \mathrm{e} . \mathrm{v} .} \frac{1}{z_{j}-\lambda}\right\rangle=\int \prod_{j=1}^{k} d \lambda_{j} \frac{1}{z_{j}-\lambda_{j}} \rho_{k}\left(\lambda_{1}, \ldots, \lambda_{k}\right) .
$$

and can be generated as in eq. (4)

$$
G_{k}\left(z_{1}, \ldots, z_{k}\right)=\left\langle\prod_{j=1}^{k} \operatorname{Tr}\left[\frac{1}{z_{j}-D}\right]\right\rangle=\left.\left(\prod_{j=1}^{k} \frac{\partial}{\partial z_{j}^{\prime}}\right)\left\langle\prod_{j=1}^{k} \frac{\operatorname{Det}\left[D-z_{j}^{\prime}\right]}{\operatorname{Det}\left[D-z_{j}\right]}\right\rangle\right|_{z_{j}^{\prime}=z_{j} \forall j}
$$

The density correlations are then given by the discontinuities with respect to all arguments (see e.g. 34]):

$$
\rho_{k}\left(\eta_{1}, \ldots, \eta_{k}\right)=\frac{1}{(2 \pi i)^{k}} \lim _{\varepsilon \rightarrow 0^{+}} \sum_{\{\sigma\}, \sigma_{j}= \pm 1}\left(\prod_{j=1}^{k} \sigma_{j}\right) G_{k}\left(\eta_{1}-i \sigma_{1} \varepsilon, \ldots, \eta_{k}-i \sigma_{k} \varepsilon\right) .
$$

In non-Hermitian theories eigenvalues are no more localised along the real (imaginary) axis, and, as a consequence, the correlation functions cannot be computed through the discontinuity of the resolvent along the support of the eigenvalues. The equation below will be used to generate the two-dimensional $\delta$-function in the complex plane instead:

$$
\partial_{z^{*}} \frac{1}{z}=\pi \delta_{\mathbb{C}}^{2}(z)
$$

Because the average in eq. (2) is now over complex eigenvalues the resolvent is well-defined everywhere in the complex plane, both in- and outside the support of $D$. From eq. (8) it follows that for the density we have

$$
\rho_{1}(z)=\frac{1}{\pi} \partial_{z^{*}} G_{1}(z) .
$$

In other words the resolvent is holomorphic outside the support, and non-holomorphic inside. As before the resolvent may be generated by differentiating the expectation value of a ratio of determinants as given in eq. (4). However, this case has an additional problem due to the fact that the microscopic field theory (such as $\epsilon \chi \mathrm{PT})$ requires the introduction of additional bosonic and fermionic conjugated quarks. This phenomenon is due to the Hermiticity requirement [37, 24, 17] for bosonic field theories to be written in terms of convergent integrals, and we will discuss that in more detail in sect. 5.1.

As before the above can be generalised to any $k$-point function, leading to the introduction of $k$ additional bosonic and fermionic quarks (or to $k$ couples of conjugated bosonic and fermionic quarks, if one needs to consider the microscopic theory), with

$$
\rho_{k}\left(z_{1}, \ldots, z_{k}\right)=\left(\prod_{j=1}^{k} \frac{1}{\pi} \partial_{z_{j}^{*}}\right) G_{k}\left(z_{1}, \ldots, z_{k}\right) .
$$


Let us mention an alternative method to generate all density correlations - apart from non-resolvent related ones: it generates the two-dimensional $\delta$-function using the identity

$$
\delta_{\mathbb{C}}^{2}(z)=\lim _{\kappa \rightarrow 0} \frac{k^{2}}{\pi\left(z \cdot z^{*}+k^{2}\right)^{2}}
$$

and hence when considering the sum of the delta functions over all the eigenvalues $\lambda$ of a non-Hermitian matrix $D$ we have (see [38]):

$$
\sum_{\lambda \in \text { e.v. }} \delta_{\mathbb{C}}^{2}(z-\lambda)=\lim _{\kappa \rightarrow 0} \frac{1}{\pi} \operatorname{Tr}\left[\frac{\kappa^{2}}{\left((z-D)(z-D)^{\dagger}+\kappa^{2}\right)^{2}}\right]
$$

\section{Zero chemical potential}

We start from the simplest case: the Hermitian chRMT corresponding to QCD with zero chemical potential. In principle this theory could be seen as a particular case of the imaginary $\mu$ case studied in sect. 4, setting $\mu=0$. Since computations are easier here, we present this case separately for pedagogic reasons.

The idea of our approach to prove the equivalence of all spectral properties in the two theories is the following: in both theories the resolvent method allows in principle to compute all $k$-point correlation function. So without taking the discontinuities, or anti-holomorphic derivatives, it is sufficient to prove that, up to an irrelevant constant, the integrals describing these generating functions of the resolvents coincide. From this knowledge follows that all the k-point correlation functions are equal, and, hence all spectral properties agree. In order to actually compute any given correlation function we may thus choose either theory, and within that theory we may even use any other equivalent method that is simpler.

Hermitian chRMT is a very efficient way to describe the spectral properties of QCD without chemical potential 1, 2]. The equivalent of the QCD Dirac operator in chRMT is given by

$$
\mathcal{D}_{f}+m_{f} \equiv\left(\begin{array}{cc}
m_{f} \mathbf{1}_{N_{+}} & i A \\
i A^{\dagger} & m_{f} \mathbf{1}_{N_{-}}
\end{array}\right), f=1, \ldots, N_{f},
$$

where $A$ is a complex $N_{+} \times N_{-}$random matrix. It describes a fixed topological sector of QCD with a topological charge $\nu=N_{+}-N_{-}$, where $\nu$ will be kept fixed while considering the $N_{+}+N_{-} \equiv N \rightarrow \infty$ limit.

Thanks to the universality of the microscopic limit of chRMT [18, 33, 19] we can choose a Gaussian weight function for the random matrix $A$. Since we are interested in finding the spectrum and the $k$-point correlation function using the resolvent method 39, 28, 29, 34, according to what was said in sect. 22 we consider the more general theory with $n_{f}$ fermionic quarks and $n_{b}$ bosonic ones and compute it's partition function:

$$
Z_{p q}=\left\langle\frac{\prod_{f}^{n_{f}} \operatorname{Det}\left[\mathcal{D}_{f}+m_{f}\right]}{\prod_{b}^{n_{b}} \operatorname{Det}\left[\mathcal{D}_{b}+m_{b}\right]}\right\rangle=\int d A e^{-\sigma N T r\left[A^{\dagger} A\right]} \frac{\prod_{f}^{n_{f}} \operatorname{Det}\left[\begin{array}{cc}
m_{f} \mathbf{1}_{N_{+}} & i A \\
i A^{\dagger} & m_{f} \mathbf{1}_{N_{-}}
\end{array}\right]}{\prod_{b}^{n_{b}} \operatorname{Det}\left[\begin{array}{cc}
m_{b} \mathbf{1}_{N_{+}} & i A \\
i A^{\dagger} & m_{b} \mathbf{1}_{N_{-}}
\end{array}\right]}
$$

Here $d A$ is the flat measure in the independent entries of the complex matrix $A$. The quantity defined above is usually called partially-quenched (pq) partition function.

In the following it will be required that $\operatorname{Re}\left(m_{b}\right)>0 \forall b$ in order to have convergent integrals generating the resolvents in the upper half-plane. The result for $\operatorname{Re}\left(m_{b}\right)<0$ may be easily recovered from the equation above 1 .

At this point we follow the common procedure [12] of writing the determinants in the numerator in terms of Gaussian fermionic integrals, and the ones in the denominator in terms of bosonic ones. We introduce two sets of $N_{+}$and $N_{-}$complex-supervector: 2 in $\left(n_{b} \mid n_{f}\right)$. In this paper we will use the boson-fermion convention

\footnotetext{
${ }^{1}$ In the literature often a diagonal matrix $S$ with elements $s_{i}= \pm 1$ is introduced to be able to work in the whole complex plane at once. For simplicity we omitted such a notation.

${ }^{2}$ The anticommuting number part of supervectors and its conjugate are independent Grassmann variables.
} 
for ordering elements in supervectors. We write these vectors in a matrix form $\psi_{g, \alpha}, \phi_{g, \beta}, \psi_{g, \alpha}^{*}$ and $\phi_{g, \beta}^{*}$ where Latin indices run over the $\left(n_{b} \mid n_{f}\right)$ superflavours and Greek indices run over the $N_{+}\left(N_{-}\right)$eigenvalues,

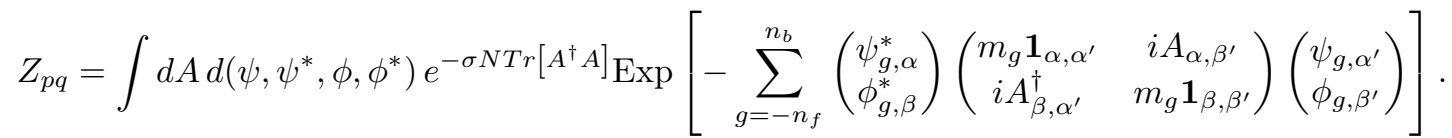

Here $d\left(\psi, \psi^{*}, \phi, \phi^{*}\right)$ is a shorthand notation for the product of the flat measures of the independent entries of the supervectors. The integral above depends on the random matrices only in a Gaussian way:

$$
\operatorname{Exp}\left[-\sigma N A_{\alpha, \beta} A_{\alpha, \beta}^{*}-i A_{\alpha, \beta}\left(\psi_{g \alpha}^{*} \phi_{g, \beta}\right)-i A_{\alpha, \beta}^{*}\left(\phi_{g, \beta}^{*} \psi_{g \alpha}\right)\right] .
$$

We can thus perform the Gaussian integration by completing the squares:

$$
\begin{aligned}
Z_{p q} \propto & \int d\left(\psi, \psi^{*}, \phi, \phi^{*}\right) \operatorname{Exp}\left[-\operatorname{Str}\left[m_{g} \cdot \sum_{\alpha} \psi_{g, \alpha} \otimes \psi_{g, \alpha}^{\dagger}+m_{g} \cdot \sum_{\beta} \phi_{g, \beta} \otimes \phi_{g, \beta}^{\dagger}\right]\right] \\
& \times \operatorname{Exp}\left[-\frac{1}{\sigma N} \operatorname{Str}\left[\sum_{\beta} \phi_{g, \beta} \otimes \phi_{h, \beta}^{\dagger} \cdot \sum_{\alpha} \psi_{h, \alpha} \otimes \psi_{g, \alpha}^{\dagger}\right]\right] .
\end{aligned}
$$

In the following we introduce the matrix notation $M_{g h}=N \cdot \delta_{g h} m_{g}$, anticipating the correct scaling later in the large- $N$ limit to obtain $N$-independent quantities in the thermodynamic limit 41. This expression is a Gaussian integral in any of the two sets of supervectors. We can easily perform one supervector Gaussian integration $\left(\phi, \phi^{\dagger}\right)$ obtaining a superdeterminant as a result:

$$
Z_{p q} \propto \int d\left(\psi, \psi^{*}\right) e^{-S \operatorname{Str}\left[m_{g} \cdot \sum_{\alpha} \psi_{g, \alpha} \otimes \psi_{g, \alpha}^{\dagger}\right]} \mathcal{S} \operatorname{det}\left[\frac{1}{N} M_{g h}+\frac{1}{\sigma N} \sum_{\alpha} \psi_{g, \alpha} \otimes \psi_{h, \alpha}^{\dagger}\right]^{-N_{-}} .
$$

The equation above depends on the supervectors only trough the sum of external products. We can now apply the superbosonisation theorem in eq. (55),

$$
\frac{1}{N_{+}} \sum_{\alpha=1}^{N_{+}} \psi_{g, \alpha} \otimes \psi_{h, \alpha}^{\dagger} \rightarrow U_{g h} \in \hat{G} l\left(n_{b} \mid n_{f}\right)
$$

where $\hat{G} l\left(n_{b} \mid n_{f}\right)$ is the maximal Riemannian submanifold of the linear group in the $\left(n_{b} \mid n_{f}\right)$ superspace [42]; matrix representations of this manifold are possible, their elements are supermatrices with $G l\left(n_{b}\right) / U\left(n_{b}\right) \oplus U\left(n_{f}\right)$ as a base manifold 3 and $2 n_{b} \cdot n_{f}$ Grassmann variables. The Haar measure on this manifold is called $d \mu_{H}(a)$ and may be expressed in terms of usual integrations trough (see eq. (69)):

$$
\int_{\hat{G} l\left(n_{b} \mid n_{f}\right)} d \mu_{H}=\int_{H_{1}=H_{1}^{\dagger}} d H_{1} \theta\left(H_{1}\right) \int_{U\left(n_{f}\right)} d \mu_{U}\left(H_{2}\right) \operatorname{Det}\left[H_{2}\right]^{n_{f}} \int d \Theta d \Theta^{\dagger} \mathcal{S} d e t\left[\begin{array}{cc}
H_{1} & \Theta^{\dagger} \\
\Theta & H_{2}
\end{array}\right]^{n_{f}-n_{b}} .
$$

Here, $d H_{1}$ is the flat measure on the Hermitian matrices, $\theta\left(H_{1}\right)$ is the product of the step function in the eigenvalues $\left(\theta\left(H_{1}\right)>0 \Longleftrightarrow H_{1}\right.$ is positive definite), $d \mu_{U}$ is the Haar measure on unitary matrices $H_{2}$ and $d \Theta, d \Theta^{\dagger}$ is the flat Grassmannian integration in the independent entries of the boson-fermion block 4 . Other parametrisations of $\hat{G l}\left(n_{b} \mid n_{f}\right)$ have been provided for some specific values of $\left(n_{b} \mid n_{f}\right)$ [29, 43, 30.

The result is $\left(N_{+}=\nu+N_{-}\right)$:

$$
\begin{aligned}
Z_{p q} & \propto \int_{\hat{G} l\left(n_{b} \mid n_{f}\right)} d \mu_{H}(U) \mathcal{S} \operatorname{det}[U]^{N_{+}} e^{-\operatorname{Str}\left[\frac{N_{+}}{N} M U\right]} \mathcal{S} \operatorname{det}\left[\sigma \frac{M}{N_{+}}+U\right]^{-N_{-}} \\
& =\int_{\hat{G} l\left(n_{b} \mid n_{f}\right)} d \mu_{H}(U) \operatorname{S} \operatorname{det}[U]^{\nu} e^{-\operatorname{Str}\left[\frac{N_{+}}{N} M U\right]} \mathcal{S} \operatorname{det}\left[1+\sigma \frac{1}{N_{+}} M \cdot U^{-1}\right]^{-N_{-}} .
\end{aligned}
$$

\footnotetext{
${ }^{3}$ The bodies, that are the complex number parts of the commuting numbers, of the boson-boson block and fermion-fermion block belong to a manifold called base manifold.

${ }^{4}$ The Grassmann variables $\Theta_{\alpha, b}$ and $\Theta_{b, \alpha}^{\dagger}$ are independent real Grassmann variables.
} 
All the equations above hold for finite $N$. As a last step we can perform the $N \rightarrow \infty$ limit. Here we send the masses $m_{h}$ to zero while keeping $M_{g h}$ fixed. Therefore the $N \rightarrow \infty$ merely leads to an expansion of the superdeterminant, without the need to perform any other approximation:

$$
\begin{aligned}
\lim _{N \rightarrow \infty} Z_{p q} & =\int_{\hat{G} l\left(n_{b} \mid n_{f}\right)} d \mu_{H}(U) \mathcal{S} \operatorname{det}[U]^{\nu} e^{S \operatorname{Str}\left[-\frac{1}{2} M U-\sigma M \cdot U^{-1}\right]} \\
& \propto \int_{\hat{G} l\left(n_{b} \mid n_{f}\right)} d \mu_{H}(U) \mathcal{S} \operatorname{det}[U]^{\nu} e^{-\sqrt{\frac{\sigma}{2}} S \operatorname{tr}\left[M \cdot U+M \cdot U^{-1}\right]} .
\end{aligned}
$$

This equation is equivalent to the partially-quenched partition function e.g. in [29], after matching parameters

$$
m_{i} N \sqrt{\frac{\sigma}{2}}=m_{i} \frac{\Sigma V}{2}
$$

We denote by $V$ the volume in $\chi \mathrm{PT}$ and by $\Sigma$ the chiral condensate, the first low energy constant in $\chi \mathrm{PT}$. Applying the resolvent method to both eq. (14) in the limit $N \rightarrow \infty$, and eq. (22) while keeping eq. (23) finite, we obtain the claimed $\epsilon \chi \mathrm{PT}$-chRMT equivalence at $\mu=0$ of all $k$-point correlation functions in the microscopic limit.

\section{Imaginary chemical potential}

The simplest generalisation of the arguments above is obtained by adding imaginary chemical potentials. This is a Hermitian theory too and, hence, the resolvent method may be applied in the very same way as before. The only difference with the former section lies in a different random matrix Dirac operator, containing one more random matrix. This model introduced in [8] follows the idea of [7] in assuming that the chemical potential term in non-diagonal in matrix space. This apparent complication by adding more random variables in fact makes the model simpler: for two different chemical potentials one can go to an eigenvalue basis and use bi-orthogonal polynomials. Below we don't need to diagonalise the matrices as we explicitly integrate them out. The equivalence between chRMT and $\epsilon \chi \mathrm{PT}$ we show thus holds for any number of different chemical potentials. However, the integrals and density correlations are so far known explicitly only when having two different chemical potentials for any number of flavours.

In principle one could also follows the idea of Stephanov 9] with only one random matrix and the $\mu$-term proportional to unity. However, for technical reasons the computation is more involved and although we don't show it here it leads to the same result.

The Dirac operator for a quark with mass $m_{f}$ and imaginary chemical potential $\mu_{f}$ is given by []]:

$$
\mathcal{D}_{f}+m_{f} \equiv\left(\begin{array}{cc}
m_{f} \mathbf{1}_{N_{+}} & i A+i \mu_{f} B \\
i A^{\dagger}+i \mu_{f} B^{\dagger} & m_{f} \mathbf{1}_{N_{-}}
\end{array}\right),
$$

and likewise for boson masses $m_{b}$ and chemical potential $\mu_{b}$. Here $A$ and $B$ are complex $N_{+} \times N_{-}$random matrices with Gaussian weights. The issue of universality is more subtle here because the matrices $A$ and $B$ will couple after changing variables. We refer to [8] for a more detailed discussion.

We will prove the equivalence of the partition functions for a generic theory with $n_{b}$ bosons and $n_{f}$ fermions. The equivalence of the spectra will then follow, applying the resolvent method. As before we will consider only bosonic masses with positive real parts,

$$
Z_{p q}=\left\langle\frac{\prod_{f}^{n_{f}} \operatorname{Det}\left[\mathcal{D}_{f}+m_{f}\right]}{\prod_{b}^{n_{b}} \operatorname{Det}\left[\mathcal{D}_{b}+m_{b}\right]}\right\rangle=\int d A d B e^{-\sigma N \operatorname{Tr}\left[A A^{\dagger}+B B^{\dagger}\right]} \frac{\prod_{f}^{n_{f}} \operatorname{Det}\left[\begin{array}{cc}
m_{f} \mathbf{1}_{N_{+}} & i A+i \mu_{f} B \\
i A^{\dagger}+i \mu_{f} B^{\dagger} & m_{f} \mathbf{1}_{N_{-}}
\end{array}\right]}{\prod_{b}^{n_{b}} \operatorname{Det}\left[\begin{array}{cc}
m_{b} \mathbf{1}_{N_{+}} & i A+i \mu_{b} B \\
i A^{\dagger}+i \mu_{f} B^{\dagger} & m_{b} \mathbf{1}_{N_{-}}
\end{array}\right]}
$$

From this point on, most of the steps are equivalent to the ones performed in the previous section: we introduce two sets of $N_{+}$and $N_{-}$complex-supervectors in $\left(n_{b} \mid n_{f}\right)$ to write the ratio of determinants as a 
Gaussian integral

$$
\begin{aligned}
& Z_{p q}=\int d A d B d\left(\psi, \psi^{*}, \phi, \phi^{*}\right) \operatorname{Exp}\left[-\sigma N\left(\operatorname{Tr}\left[A^{\dagger} A\right]+\operatorname{Tr}\left[B^{\dagger} B\right]\right)\right] \\
& \times \operatorname{Exp}\left[-\sum_{g=-n_{f}}^{n_{b}}\left(\begin{array}{c}
\psi_{g, \alpha}^{*} \\
\phi_{g, \beta}^{*}
\end{array}\right)\left(\begin{array}{cc}
m_{g} \mathbf{1}_{\alpha, \alpha^{\prime}} & \left(i A+i \mu_{g} B\right)_{\alpha, \beta^{\prime}} \\
\left(i A^{\dagger}+i \mu_{g} B^{\dagger}\right)_{\beta, \alpha^{\prime}} & m_{g} \mathbf{1}_{\beta, \beta^{\prime}}
\end{array}\right)\left(\begin{array}{l}
\psi_{g, \alpha^{\prime}} \\
\phi_{g, \beta^{\prime}}
\end{array}\right)\right] .
\end{aligned}
$$

We reduce all the dependence on random matrices in terms of Gaussian functions:

$$
\begin{aligned}
& \operatorname{Exp}\left[-\sigma N A_{\alpha, \beta} A_{\alpha, \beta}^{*}-i A_{\alpha, \beta}\left(\psi_{g \alpha}^{*} \phi_{g, \beta}\right)-i A_{\alpha, \beta}^{*}\left(\phi_{g, \beta}^{*} \psi_{g \alpha}\right)\right] \\
& \times \operatorname{Exp}\left[-\sigma N B_{\alpha, \beta} B_{\alpha, \beta}^{*}-i \mu_{g} B_{\alpha, \beta}\left(\psi_{g \alpha}^{*} \phi_{g, \beta}\right)-i \mu_{g} B_{\alpha, \beta}^{*}\left(\phi_{g, \beta}^{*} \psi_{g \alpha}\right)\right] .
\end{aligned}
$$

Next we can perform the Gaussian integration completing the squares:

$$
\begin{aligned}
& Z_{p q} \propto \int d\left(\psi, \psi^{*}, \phi, \phi^{*}\right) \operatorname{Exp}\left[-\operatorname{Str}\left[m_{g} \cdot \sum_{\alpha} \psi_{g, \alpha} \otimes \psi_{g, \alpha}^{\dagger}+m_{g} \cdot \sum_{\beta} \phi_{g, \beta} \otimes \phi_{g, \beta}^{\dagger}\right]\right] \\
& \times \operatorname{Exp}\left[-\frac{1}{\sigma N} \operatorname{Str}\left[\sum_{\beta} \phi_{g, \beta} \otimes \phi_{h, \beta}^{\dagger} \cdot \sum_{\alpha} \psi_{h, \alpha} \otimes \psi_{g, \alpha}^{\dagger}\right]\right] \\
& \times \operatorname{Exp}\left[-\frac{1}{\sigma N} \operatorname{Str}\left[\mu_{g} \cdot \sum_{\beta} \phi_{g, \beta} \otimes \phi_{h, \beta}^{\dagger} \cdot \mu_{h} \cdot \sum_{\alpha} \psi_{h, \alpha} \otimes \psi_{g, \alpha}^{\dagger}\right]\right] .
\end{aligned}
$$

Here we introduce again the matrix notation $M_{g h} \equiv N \cdot \delta_{g h} m_{g}$, as well as $B_{g h}^{(\mu)}=\sqrt{\frac{N}{2}} \delta_{g h} \mu_{g}$, anticipating also the proper scaling of $\mu$ with $N$ below. This expression is a Gaussian integral in any of the two sets of supervectors. As before we explicitly integrate one set of supervectors and express the remaining external product in terms of an integration over $\hat{G} l\left(n_{b} \mid n_{f}\right)$. The result is:

$$
Z_{p q} \propto \int d\left(\psi, \psi^{*}\right) e^{-\operatorname{Str}\left[\frac{1}{N} M \cdot \sum_{\alpha} \psi_{\alpha} \otimes \psi_{\alpha}^{\dagger}\right]} \mathcal{S} d e t\left[\frac{1}{N} M+\frac{1}{\sigma N} \sum_{\alpha} \psi_{\alpha} \otimes \psi_{\alpha}^{\dagger}+\frac{2}{\sigma N^{2}} B^{(\mu)} \cdot \sum_{\alpha} \psi_{\alpha} \otimes \psi_{\alpha}^{\dagger} \cdot B^{(\mu)}\right]^{-N_{-}} .
$$

We can use again the theorem of section 6 obtaining:

$$
Z_{p q} \propto \int_{\hat{G} l\left(n_{b} \mid n_{f}\right)} d \mu_{H}(U) \mathcal{S} \operatorname{det}[U]^{\nu} e^{-S t r\left[\frac{N_{+}}{N} M U\right]} \mathcal{S} \operatorname{det}\left[1+\sigma \frac{1}{N_{+}} M \cdot U^{-1}+\frac{2}{N} B^{(\mu)} U B^{(\mu)} U^{-1}\right]^{-N_{-}} .
$$

This result is again exact for any finite $N$. If we now take the large- $N$ limit while keeping $M$ and $B^{(\mu)}$ fixed we obtain finally:

$$
\lim _{N \rightarrow \infty} Z_{p q}=\int_{\hat{G} l\left(n_{b} \mid n_{f}\right)} d \mu_{H}(U) \mathcal{S} \operatorname{det}[U]^{\nu} e^{-\sqrt{\frac{\sigma}{2}} S \operatorname{tr}\left[M \cdot U+M \cdot U^{-1}\right]-\operatorname{Str}\left[B^{(\mu)} U B^{(\mu)} U^{-1}\right]}
$$

where our only approximation has been the expansion of the superdeterminant.

This expression is equivalent to the $\epsilon \chi \mathrm{PT}$ effective partition [31, where in order to match we use the following relations [26, 3]

$$
\begin{aligned}
m_{i} N \sqrt{\frac{\sigma}{2}} & =m_{i} \frac{\Sigma V}{2}, \\
\mu_{i} \sqrt{N} & =\mu_{D_{i}} F_{\pi} \sqrt{V} .
\end{aligned}
$$

Here $\mu_{D}$ is a dimensional constant, instead of the $\mu$ used above that is dimensionless. The additional parameter $F_{\pi}$ is the pion decay constant, the second low energy constant in the leading order chiral Lagrangian. Thus we have established the $\epsilon \chi \mathrm{PT}$-chRMT equivalence for all $k$-point correlation functions with imaginary chemical potential.

\footnotetext{
${ }^{5}$ In the theory with real chemical potential in the next section this limit is called weak non-Hermiticity limit 44 . While we inherit the same scaling here our operators are always Hermitian.
} 


\section{Real chemical potential}

Before starting the proof for real chemical potential it's better to spend a few words on the definition of theories with bosonic quarks. It may help understanding both the result and the proof.

\subsection{Bosonic partition function with $\mu \neq 0$}

The sign problem makes it impossible to write the inverse Dirac operator as a convergent bosonic integral: Hermitisation is needed [45, 46] and a new particle content of the theory comes out [37, 24, 17.

Let's consider the simplest example, $n_{b}=1, n_{f}=0$, following [17. The partition function is given by:

$$
\left\langle\frac{1}{\operatorname{Det}\left[D+\mu \gamma_{0}+m\right]}\right\rangle \text {. }
$$

In the microscopic theory (QCD) the inverse power of the determinant should be drawn from the integration of bosonic quarks. This integration is well defined only if the integral is convergent, that is, if the Hermitian part of the operator is positive (or negative) defined; this requirement is fulfilled when Dirac operator is given by a non-Hermitian (kinetic) part plus a mass term (proportional to the identity operator), but this is not the case for a Dirac operator suffering from the sign problem.

The way to bypass this problem is to add an additional bosonic conjugate quark and a fermionic one with mass $-m^{*}$ and chemical potential $-\mu$, respectively,

$$
\begin{aligned}
\left\langle\frac{1}{\operatorname{Det}\left[D+\mu \gamma_{0}+m\right]}\right\rangle & =\left\langle\frac{\operatorname{Det}\left[D-\mu \gamma_{0}-m^{*}\right]}{\operatorname{Det}\left[D+\mu \gamma_{0}+m\right] \operatorname{Det}\left[D-\mu \gamma_{0}-m^{*}\right]}\right\rangle \\
& \propto\left\langle\frac{\operatorname{Det}\left[D-\mu \gamma_{0}-m^{*}\right]}{\operatorname{Det}\left[\begin{array}{cc}
0 & \left.D+\mu \gamma_{0}+m\right] \\
D-\mu \gamma_{0}-m^{*} & 0
\end{array}\right]} .\right.
\end{aligned}
$$

The operator in the denominator is now anti-Hermitian and hence has only pure imaginary eigenvalues. If we consider the matrix

$$
\left(\begin{array}{cc}
\varepsilon & D+\mu \gamma_{0}+m \\
D-\mu \gamma_{0}-m^{*} & \varepsilon
\end{array}\right)
$$

(sending $\varepsilon \rightarrow 0$ at the end) we have an operator fulfilling the positivity requirements to have a properly defined theory. This process is usually called Hermitisation [45, 46], and although it could seem as just a mathematical trick this is not the case: the possibility of writing the inverse determinants as convergent integrals is a feature of the underlying theory. This procedure modifies the form of the mass term in $\epsilon \chi \mathrm{PT}[24$, 17, and for the most general result we refer to eq. (49), where the generalisation to any number $n_{b}$ of bosonic uncoupled quarks is straightforward: $\left(N_{b} \mid 0\right) \rightarrow\left(N_{b}+N_{b}^{*} \mid N_{b}^{*}\right)$. Despite this regularisation is a feature of the microscopic underlying theory, it may happen that we have, in an effective theory, properly defined integrals even without it, and that no such Hermitisation is needed. This is the case of non-Hermitian Random Matrix Theory with uncoupled bosons [47, 16, 17.

\subsection{Equivalence for real chemical potential}

As already hinted in sect. 2 and explained in sect. 5.1 the resolvent method may require the Hermitisation of the boson-boson part of Dirac operator 37]:

$$
\begin{aligned}
Z_{p q} & =\left\langle\frac{\prod_{i}^{n_{v}} \operatorname{Det}\left[D_{i}+z_{i}\right]}{\prod_{i}^{n_{v}} \operatorname{Det}\left[D_{i}+\tilde{z}_{i}\right]} \prod_{f}^{N_{f}} \operatorname{Det}\left[D_{f}+m_{f}\right]\right\rangle \\
& =\left\langle\frac{\prod_{i}^{n_{v}} \operatorname{Det}\left[D_{i}+z_{i}\right] \operatorname{Det}\left[D_{i}+\tilde{z}_{i}\right]^{*}}{\prod_{i}^{n_{v}} \operatorname{Det}\left[D_{i}+\tilde{z}_{i}\right] \operatorname{Det}\left[D_{i}+\tilde{z}_{i}\right]^{*}} \prod_{f}^{N_{f}} \operatorname{Det}\left[D_{f}+m_{f}\right]\right\rangle \\
& =\lim _{\varepsilon \rightarrow 0}\left\langle\frac{\prod_{i}^{n_{v}} \operatorname{Det}\left[D_{i}+z_{i}\right] \operatorname{Det}\left[D_{i}+\tilde{z}_{i}\right]^{*}}{\prod_{i}^{n_{v}} \operatorname{Det}\left[\left(D_{i}+\tilde{z}_{i}\right)\left(D_{i}^{\dagger}+\tilde{z}_{i}^{*}\right)+\varepsilon^{2}\right]} \prod_{f}^{N_{f}} \operatorname{Det}\left[D_{f}+m_{f}\right]\right\rangle .
\end{aligned}
$$


The first line is the proper definition of the resolvent, the second, that is obtained from the first by a trivial step, is the Hermitised version, the last line is the regularised integral. Hermitisation is necessary both for the computation below and for the underlying microscopic theory, but it's not always necessary for making the computation in RMT [47, 17].

In the second line the additional valence quarks are in conjugate pairs $\left(n_{b}+n_{b}^{*} \mid n_{v}+n_{v}^{*}+N_{f}\right)$ (the replacement $\tilde{z}_{i} \rightarrow z_{i}$ in the numerator is irrelevant). It will be sufficient to consider the conjugate fermionic quarks as independent quarks with mass $-m^{*}$ and chemical potential $-\mu$; concerning the bosonic conjugate quark, as already seen in sect. 5.1, there's a deep reason that forces us to treat them simultaneously.

We mention in passing that a purely bosonic theory with bosons in conjugated pairs $\left(n_{b}+n_{b}^{*}, 0\right)$ occurs when applying the replica trick or Toda lattice equation [9, 16, 24, 17].

In the following we will prove the equivalence of the partition functions of $\epsilon \chi \mathrm{PT}$ and chRMT at non zero chemical potential with $n_{f}$ fermionic quarks with given masses and baryonic potential $\left(m_{f}, \mu_{f}\right)$, and $n_{b}$ couples of conjugated bosonic quarks, with parameters $\left(m_{b}, \mu_{b}\right)$ and $\left(-m_{b}^{*},-\mu_{b}\right)$. The partially quenched theory with $N_{f}$ physical quarks, $n_{v}$ couples of conjugated fermionic quarks and $n_{v}$ couples of bosonic quarks will result as a special case. The equivalence of the spectra follows applying the resolvent method in both theories.

We will again use the the two-matrix model as was introduced by Osborn [7], but the same calculation can be done also for the Stephanov model. The equivalent of the QCD Dirac operator for a quark with mass $m_{f}$ and chemical potential $\mu_{f}$ is given by:

$$
\mathcal{D}_{f}+m_{f} \equiv\left(\begin{array}{cc}
m_{f} \mathbf{1}_{N_{+}} & i A+\mu_{f} B \\
i A^{\dagger}+\mu_{f} B^{\dagger} & m_{f} \mathbf{1}_{N_{-}}
\end{array}\right) .
$$

where $A$ and $B$ are complex $N_{+} \times N_{-}$random matrices.

The partition function equivalent to partially quenched QCD is:

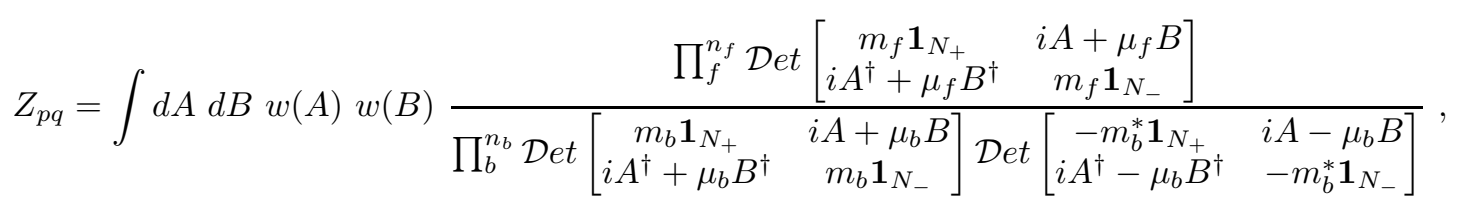

with a Gaussian weight function

$$
w(X)=\operatorname{Exp}\left[-\sigma N \operatorname{Tr}\left[X^{\dagger} X\right]\right]
$$

In order to write the inverse determinants as bosonic Gaussian integrals we perform the same anti-Hermitisation as in eq. (34), obtaining an anti-Hermitian matrix apart from an $\varepsilon$ times the identity. The regularised denominator is:

$$
\begin{aligned}
&(-)^{N_{+}}-N_{-} \mathcal{D} e t\left[\left(\begin{array}{ccc}
m_{b} \mathbf{1}_{N_{+}} & i A+\mu_{b} B \\
i A^{\dagger}+\mu_{b} B^{\dagger} & m_{b} \mathbf{1}_{N_{-}}
\end{array}\right) \cdot\left(\begin{array}{cc}
-m_{b}^{*} \mathbf{1}_{N_{+}} & i A-\mu_{b} B \\
i A^{\dagger}-\mu_{b} B^{\dagger} & -m_{b}^{*} \mathbf{1}_{N_{-}}
\end{array}\right)-\varepsilon^{2} \mathbf{1}_{N_{+}+N_{-}}\right]= \\
&=\operatorname{Det}\left[\left(\begin{array}{cccc}
\varepsilon \mathbf{1}_{N_{+}} & 0 & m_{b} \mathbf{1}_{N_{+}} & i A+\mu_{b} B \\
0 & \varepsilon \mathbf{1}_{N_{-}} & i A^{\dagger}+\mu_{b} B^{\dagger} & m_{b} \mathbf{1}_{N_{-}} \\
-m_{b}^{*} \mathbf{1}_{N_{+}} & i A-\mu_{b} B & \varepsilon \mathbf{1}_{N_{+}} & 0 \\
i A^{\dagger}-\mu_{b} B^{\dagger} & -m_{b}^{*} \mathbf{1}_{N_{-}} & 0 & \varepsilon \mathbf{1}_{N_{-}}
\end{array}\right)\right] \\
&=\operatorname{Det}\left[\left(\begin{array}{cccc}
\epsilon+\frac{m-m^{*}}{2} & i A & \frac{m+m^{*}}{2} & \mu B \\
i A^{\dagger} & \epsilon+\frac{m-m^{*}}{2} & \mu B^{\dagger} & \frac{m+m^{*}}{2} \\
-\frac{m+m^{*}}{2} & -\mu B & \epsilon-\frac{m-m^{*}}{2} & -i A \\
-\mu B^{\dagger} & -\frac{m+m^{*}}{2} & -i A^{\dagger} & \epsilon-\frac{m-m^{*}}{2}
\end{array}\right)\right] .
\end{aligned}
$$

We introduce two sets of $N_{+}$and $N_{-}$complex supervectors in $\left(2 n_{b} \mid n_{f}\right)$ to write the ratio of determinants 
as a Gaussian integral

$$
\begin{aligned}
& Z_{p q}=\int d A d B d\left(\psi, \psi^{*}, \phi, \phi^{*}\right) \operatorname{Exp}\left[-\sigma N\left(\operatorname{Tr}\left[A^{\dagger} A\right]+\operatorname{Tr}\left[B^{\dagger} B\right]\right)\right] \\
& \times \operatorname{Exp}\left[-\sum_{f}^{n_{f}}\left(\begin{array}{c}
\psi_{f, \alpha}^{*} \\
\phi_{f, \beta}^{*}
\end{array}\right)\left(\begin{array}{cc}
m_{f} \mathbf{1}_{\alpha, \alpha^{\prime}} & \left(i A+\mu_{f} B\right)_{\alpha, \beta^{\prime}} \\
\left(i A^{\dagger}+\mu_{f} B^{\dagger}\right)_{\beta, \alpha^{\prime}} & m_{f} \mathbf{1}_{\beta, \beta^{\prime}}
\end{array}\right)\left(\begin{array}{l}
\psi_{f, \alpha^{\prime}} \\
\phi_{f, \beta^{\prime}}
\end{array}\right)\right] \\
& \times \operatorname{Exp}\left[-\sum_{b}^{n_{b}}\left(\begin{array}{c}
\psi_{2 b-1, \alpha}^{*} \\
\phi_{2 b-1, \beta}^{*} \\
\psi_{2 b, \alpha}^{*} \\
\phi_{2 b, \beta}^{*}
\end{array}\right)\left(\begin{array}{cccc}
\epsilon+\frac{m_{b}-m_{b}^{*}}{2} & i A_{\alpha \beta^{\prime}} & \frac{m_{b}+m_{b}^{*}}{2} & \mu_{b} B_{\alpha \beta^{\prime}} \\
i A_{\beta \alpha^{\prime}}^{\dagger} & \epsilon+\frac{m_{b}-m_{b}^{*}}{2} & \mu_{b} B_{\beta \alpha^{\prime}}^{\dagger} & \frac{m_{b}+m_{b}^{*}}{2} \\
-\frac{m_{b}+m_{b}^{*}}{2} & -\mu_{b} B_{\alpha \beta^{\prime}} & \epsilon-\frac{m_{b}-m_{b}^{*}}{2} & -i A_{\alpha \beta^{\prime}} \\
-\mu_{b}^{*} B_{\beta \alpha^{\prime}}^{\dagger} & -\frac{m_{b}+m_{b}^{*}}{2} & -i A_{\beta \alpha^{\prime}}^{\dagger} & \epsilon-\frac{m_{b}-m_{b}^{*}}{2}
\end{array}\right)\left(\begin{array}{c}
\psi_{2 b-1, \alpha^{\prime}} \\
\phi_{2 b-1, \beta^{\prime}} \\
\psi_{2 b, \alpha^{\prime}} \\
\phi_{2 b, \beta^{\prime}}
\end{array}\right)\right] .
\end{aligned}
$$

As before we have reduced all the dependence on random matrices in terms of Gaussian functions:

$$
\begin{aligned}
& \operatorname{Exp}\left[-\sigma N A_{\alpha, \beta} A_{\alpha, \beta}^{*}-i A_{\alpha, \beta}\left(\sum_{f} \psi_{f, \alpha}^{*} \phi_{f, \beta}+\sum_{b}\left(\psi_{2 b-1, \alpha}^{*} \phi_{2 b-1, \beta}-\psi_{2 b, \alpha}^{*} \phi_{2 b, \beta}\right)\right)\right. \\
& \left.-i A_{\alpha, \beta}^{*}\left(\sum_{f} \phi_{f, \beta}^{*} \psi_{f, \alpha}+\sum_{b}\left(\phi_{2 b-1, \beta}^{*} \psi_{2 b-1, \alpha}-\phi_{2 b, \beta}^{*} \psi_{2 b, \alpha}\right)\right)\right] \\
& \times \operatorname{Exp}\left[-\sigma N B_{\alpha, \beta} B_{\alpha, \beta}^{*}-B_{\alpha, \beta}\left(\sum_{f} \mu_{f} \psi_{f, \alpha}^{*} \phi_{f, \beta}+\sum_{b} \mu_{b}\left(\psi_{2 b-1, \alpha}^{*} \phi_{2 b, \beta}-\psi_{2 b, \alpha}^{*} \phi_{2 b-1, \beta}\right)\right)\right. \\
& \left.-B_{\alpha, \beta}^{*}\left(\sum_{f} \mu_{f} \phi_{f, \beta}^{*} \psi_{f, \alpha}+\sum_{b} \mu_{b}\left(-\phi_{2 b, \beta}^{*} \psi_{2 b-1, \alpha}+\phi_{2 b-1, \beta}^{*} \psi_{2 b, \alpha}\right)\right)\right],
\end{aligned}
$$

and we can perform the Gaussian integration completing the squares:

$$
\begin{aligned}
& \operatorname{Exp}\left[-\frac{1}{\sigma N} \sum_{\alpha, \beta} \psi_{g, \alpha}^{*} \Gamma_{g l}^{A} \phi_{l, \beta} \phi_{m, \beta}^{*} \Gamma_{m, n}^{A} \psi_{n, \alpha}+\frac{2}{\sigma N^{2}} \sum_{\alpha, \beta} \psi_{g, \alpha}^{*} \Gamma_{g l}^{B} \phi_{l, \beta} \phi_{m, \beta}^{*} \Gamma_{m, n}^{B} \psi_{n, \alpha}\right]= \\
= & \operatorname{Exp}\left[-\frac{1}{\sigma N} \operatorname{Str}\left[\Gamma^{A} \cdot \sum_{\alpha}\left(\phi_{\alpha} \otimes \phi_{\alpha}^{\dagger}\right) \cdot \Gamma^{A} \cdot \sum_{\beta}\left(\psi_{\beta} \otimes \psi_{\beta}^{\dagger}\right)-\frac{2}{N} \Gamma^{B} \cdot \sum_{\alpha}\left(\phi_{\alpha} \otimes \phi_{\alpha}^{\dagger}\right) \cdot \Gamma^{B} \cdot \sum_{\beta}\left(\psi_{\beta} \otimes \psi_{\beta}^{\dagger}\right)\right]\right]
\end{aligned}
$$

Here we have introduced the $\left(2 n_{b} \mid n_{f}\right) \times\left(2 n_{b} \mid n_{f}\right)$ supermatrices

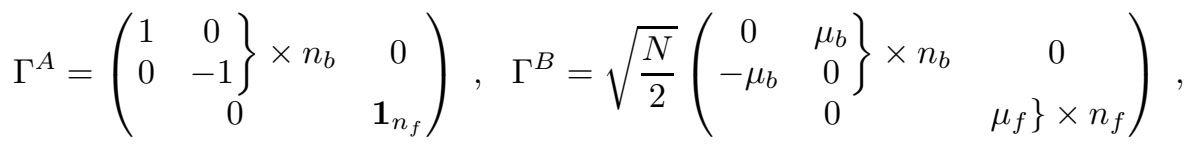

and the mass matrix:

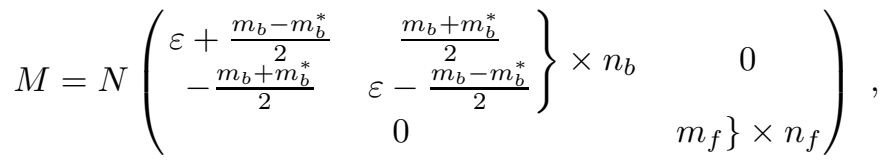

anticipating their $N$-dependence below. We can rewrite the partition function (40):

$$
\begin{aligned}
Z_{p q}= & \left.\int d\left(\psi, \psi^{*} \phi, \phi^{*}\right) \operatorname{Exp}\left[-S t r\left[\frac{1}{N} M \cdot \sum_{\beta} \psi_{\beta} \otimes \psi_{\beta}^{\dagger}\right]\right]\right] \\
& \times \operatorname{Exp}\left[-\operatorname{Str}\left[\sum_{\alpha} \phi_{\alpha} \otimes \phi_{\alpha}^{\dagger} \cdot\left(\frac{1}{N} M+\frac{1}{\sigma N} \Gamma^{A} \cdot \sum_{\beta} \psi_{\beta} \otimes \psi_{\beta}^{\dagger} \cdot \Gamma^{A}-\frac{2}{\sigma N^{2}} \Gamma^{B} \cdot \sum_{\beta} \psi_{\beta} \otimes \psi_{\beta}^{\dagger} \cdot \Gamma^{B}\right)\right]\right] .
\end{aligned}
$$


From this point on the procedure is the same as before: we integrate explicitly the sets of supervectors $\phi, \phi^{*}$ and use the superbosonisation theorem

$$
\begin{aligned}
Z_{p q}= & \int d\left(\psi, \psi^{*}\right) \operatorname{Exp}\left[-\operatorname{Str}\left[\frac{1}{N} M \cdot \sum_{\beta} \psi_{\beta} \otimes \psi_{\beta}^{\dagger}\right]\right] \\
& \times \mathcal{S} \operatorname{det}\left[\frac{1}{N} M+\frac{1}{\sigma N} \Gamma^{A} \sum_{\beta} \psi_{\beta} \otimes \psi_{\beta}^{\dagger} \Gamma^{A}-\frac{2}{\sigma N^{2}} \Gamma^{B} \sum_{\beta} \psi_{\beta} \otimes \psi_{\beta}^{\dagger} \Gamma^{B}\right]^{-N_{-}} \\
\propto & \int_{\hat{G} l\left(2 n_{b} \mid n_{f}\right)} d \mu_{H}(U) \mathcal{S} \operatorname{det}[U]^{N_{+}} e^{-\operatorname{Str}\left[\frac{N_{+}}{N} M \cdot U\right]} \operatorname{S} \operatorname{det}\left[\frac{1}{N} M+\frac{N_{+}}{\sigma N} \Gamma^{A} \cdot U \cdot \Gamma^{A}-\frac{2 N_{+}}{\sigma N^{2}} \Gamma^{B} \cdot U^{B} \Gamma^{B}\right]^{-N_{-}} \cdot
\end{aligned}
$$

This result is valid for finite $N$. Once performing the $N \rightarrow \infty$ weak non-Hermiticity limit [44, keeping $M$ fixed as well as $\Gamma^{B}$, the following result is obtained

$$
\lim _{N \rightarrow \infty} Z_{p q}=\int_{\hat{G} l\left(2 n_{b} \mid n_{f}\right)} d \mu_{H}(U) \mathcal{S} \operatorname{det}[U]^{\nu} \operatorname{Exp}\left[S t r\left[-\sqrt{\frac{\sigma}{2}} M\left(U+\Gamma^{A} \cdot U^{-1} \cdot \Gamma^{A}\right)+\Gamma^{A} \Gamma^{B} U \Gamma^{B} \Gamma^{A} U^{-1}\right]\right] .
$$

Rotating all the matrices under the superunitary transformation $X \rightarrow T^{\dagger} \cdot X \cdot T$ with

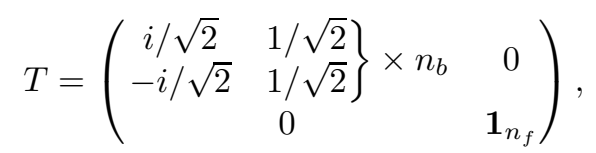

we obtain the new result for Hermitised $\epsilon \chi \mathrm{PT}$ with real $\mu \neq 0$, generalising previous results in the literature [24, 17]:

$$
\lim _{N \rightarrow \infty} Z_{p q}=\int_{\hat{G} l\left(2 n_{b} \mid n_{f}\right)} d \mu_{H}(U) \mathcal{S} \operatorname{det}[U]^{\nu} \operatorname{Exp}\left[S t r\left[-\sqrt{\frac{\sigma}{2}} \hat{M}\left(U+I \cdot U^{-1} \cdot I\right)+B_{+}^{(\mu)} U B_{-}^{(\mu)} U^{-1}\right]\right]
$$

where

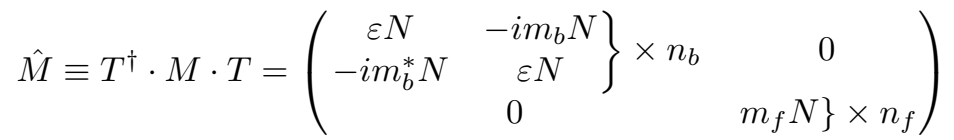

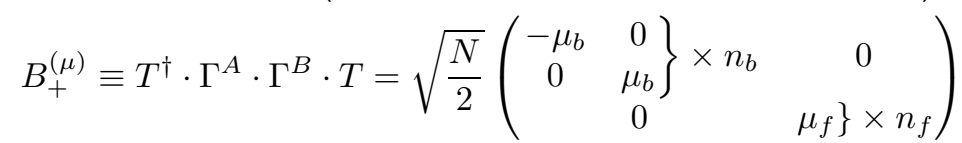

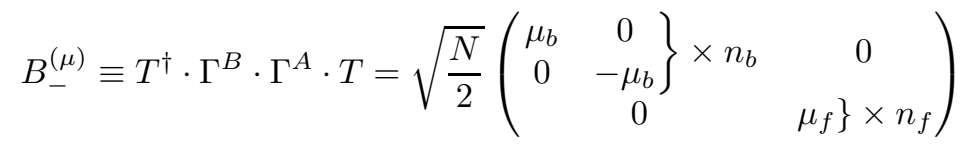

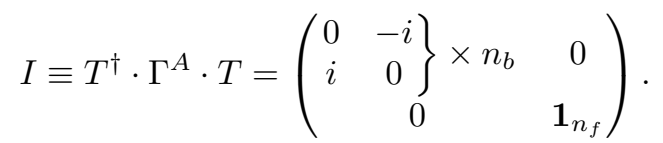

It is expressed in term of $\Sigma, F_{\pi}$ and chemical potential using eq. (31). For details on the Haar measure $d \mu_{H}(U)$ we refer to the next section.

The expression above is a generalisation of $\epsilon \chi \mathrm{PT}$ with one pair of bosons [24, 17, and of $\epsilon \chi \mathrm{PT}$ with $N_{f}$ fermions $\epsilon \chi \mathrm{PT}$ [35]. The signature of the boson-boson block in the metric $I$ differs from 24] by a factor of $i$ because we have used an anti-Hermitisation here, see subsection 5.1 instead of a Hermitisation there. The main point is that the two mass terms have different signs if considering bosonic or fermionic quarks, see eqs. (102) and (131) of [24], respectively.

The particular cases of eq. (49) already known in literature 24, 17, 35, have been derived starting from the symmetries of the microscopic theory under vector and axial transformations too, and we suppose that the same 
arguments can be applied to the general case. The existence of two different matrices $B_{ \pm}^{(\mu)}$ is due to the fact that the covariant derivative has a different behaviour on bosonic and fermionic quarks in $\epsilon \chi \mathrm{PT}$ as explained in [17.

\section{Superbosonisation theorem}

The aim of this section is to find a way to express integrals of functions of external products of supervectors in terms of integrals over a smaller space. In formulas we are going to study

$$
\int d\left(\psi, \psi^{*}\right) f\left(\sum_{k} \psi_{k} \otimes \psi_{k}^{\dagger}\right)
$$

where $\psi_{k}, \psi_{k}^{\dagger}$ are complex vectors of $n_{b}$ bosons and $n_{f}$ fermions, $d\left(\psi, \psi^{*}\right)$ is a shorthand notation for $\prod_{k}^{N} d \psi_{k} d \psi_{k}^{*}$ and $f$ is a function defined over $\left(n_{b} \mid n_{f}\right)$ supermatrices.

The main idea is to embed $\sum_{k} \psi_{k} \otimes \psi_{k}^{\dagger}$ in some manifold where we can define a $\delta$-function and where computations are feasible. At a merely symbolic level we have:

$$
\int d\left(\psi, \psi^{*}\right) f\left(\sum_{k} \psi_{k} \otimes \psi_{k}^{\dagger}\right)=\int_{\mathcal{M}} d a f(a) \int d\left(\psi, \psi^{*}\right) \delta_{\mathcal{M}}\left(a-\sum_{k} \psi_{k} \otimes \psi_{k}^{\dagger}\right) .
$$

In order to give a meaning to the equation above we have to specify which is the manifold $\mathcal{M}$ we use and which is the measure we use on it (the $\delta$-function depends on it). The use of a $\delta$-function requires $f$ to be continuous with respect to the metric chosen.

A good choice is to take as $\mathcal{M}$ in eq. (52) the manifold of super-Hermitian matrices. One could ask whether or not a $\delta$-function may be used dealing with matrices like external product of supervectors [11] in appendix A we show that such a $\delta$ may be properly defined. The $\delta$-function may be written using an extension of the usual Fourier-transform representation of the $\delta$-function on Hermitian matrices (see appendix A).

A crucial ingredient in this proof will be the possibility to flip the order of integration. Changing the order between commuting and anti-commuting variables is not a problem, but problems can arise when considering the case of two commuting variables. In order to see where these problems come from, and how to avoid them, let's consider a simple example, the one of a single commuting number vector of length 1 :

$$
\begin{aligned}
\int_{\mathbb{C}} d^{2} z f\left(z \cdot z^{*}\right) & =\int_{\mathbb{C}} d^{2} z \int_{\mathbb{R}} d x f(x) \delta\left(x-z z^{*}\right) \\
& =\int_{\mathbb{R}} d x f(x) \int_{\mathbb{C}} d^{2} z \delta\left(x-z z^{*}\right) \\
& =\int_{\mathbb{R}} d x f(x) \int_{\mathbb{C}} d^{2} z \int_{\mathbb{R}} d y e^{i y\left(x-z z^{*}\right)}
\end{aligned}
$$

The first change of variable is always allowed when considering converging integrals. At this point we need to flip the order of integration of $y$ and $z, z^{*}$, and this is an illicit step since the integrals are not converging. This problem may be avoided considering a real quantity $\eta>0$

$$
\begin{aligned}
\int_{\mathbb{C}} d^{2} z f\left(z z^{*}\right) & =\int_{\mathbb{C}} d^{2} z f\left(z z^{*}\right) e^{(\eta-\eta) z z^{*}} \\
& =\int_{\mathbb{R}} d x f(x) e^{\eta x} \int_{\mathbb{C}} d^{2} z \int_{\mathbb{R}} \frac{d y}{2 \pi} e^{i y\left(x-z z^{*}\right)} e^{-\eta z z^{*}} \\
& =\int_{\mathbb{R}} d x f(x) e^{\eta x} \int_{\mathbb{R}} \frac{d y}{2 \pi} e^{i y x} \int_{\mathbb{C}} d^{2} z e^{-(\eta+i y) z z^{*}} \\
& =\int_{\mathbb{R}} d x f(x) e^{\eta x} \int_{\mathbb{R}} \frac{d y}{2 \pi} e^{i y x} \frac{(-i) \pi}{y-i \eta} \\
& =\int_{\mathbb{R}} d x f(x) e^{\eta x} e^{-\eta x} \pi \theta(x)=\pi \int_{0}^{\infty} d x f(x)
\end{aligned}
$$


and all the steps are mathematically rigorous any time we can apply a $\delta$-distribution as a functional to the function $f(x) e^{\eta x}$. The symbol $\theta(x)$ indicates the step function.

This easy example is conceptually not too different from the proof of the following theorem.

Theorem 1 (Superbosonisation) Let $f$ be a function defined on the $\left(n_{b} \mid n_{f}\right)$ supermatrices, then the following identity holds

$$
\int \prod_{k}^{N} d \psi_{k} d \psi_{k}^{*} f\left(\sum_{k} \psi_{k} \otimes \psi_{k}^{\dagger}\right) \propto \int_{\hat{G} l\left(n_{b} \mid n_{f}\right)} d \mu_{H}(U) \operatorname{Sdet}[U]^{N} f(U)
$$

whenever the integral on the l.h.s. is well defined. The first integration is performed over $N$ complex supervectors in $\left(n_{b} \mid n_{f}\right)$ and $d \mu_{H}(U)$ denotes the Haar measure over $\hat{G l}\left(n_{b} \mid n_{f}\right)$.

The idea of expressing integrals of a function of an external product $\sum_{k} \psi_{k} \otimes \psi_{k}^{\dagger}$ in terms of an integral of the same function over a simpler space has been widely used in physics: it was derived for the first time in 48 for external products of Grassmannian vectors appearing in exponential function (the term "bosonisation" comes from this kind of application), subsequently in [27. for commuting number vectors and in [49, 50, 51, 52, 53. for supervectors. This (super)bosonisation may be seen as application of the Riesz Representation Theorem 6 . However, up to our knowledge, a graded version of the last theorem is lacking.

By coincidence the superbosonisation theorem has been independently developed at the very same time 7 of an analogous theorem on superbosonisation by Littelmann, Sommers and Zirnbauer [10, 11]. Anyway the proof they give is different from ours, theirs is an algebraic proof, ours uses analysis instruments. The main difference is that we base our proof on the existence of a $\delta$-function fulfilling eq. (52), and that collaboration develops a powerful apparatus in order not to use such an equation [10. For this reason we have chosen to show in appendix $\mathrm{A}$ all the details concerning the mathematical rigorousness of our definition.

Proof: As already hinted above we write the l.h.s. of eq. (55) introducing an additional integration over super-Hermitian matrices:

$$
\begin{aligned}
\int d\left(\psi, \psi^{*}\right) f\left(\sum_{k} \psi_{k} \otimes \psi_{k}^{\dagger}\right) & =\int_{H=H^{\dagger}} d H f(H) e^{\eta \operatorname{Str}[H]} \int d\left(\psi, \psi^{*}\right) \delta\left(\sum_{k} \psi_{k} \otimes \psi_{k}^{\dagger}-H\right) e^{-\eta \sum_{k} \psi_{k}^{\dagger} \cdot \psi_{k}} \\
& \propto \int_{H=H^{\dagger}} d H f(H) e^{\eta \operatorname{Str}[H]} \int d\left(\psi, \psi^{*}\right) e^{-\eta \sum_{k} \psi_{k}^{\dagger} \cdot \psi_{k}} \int_{F=F^{\dagger}} d F e^{i F\left(H-\sum_{k} \psi_{k} \otimes \psi_{k}^{\dagger}\right)} \\
& =\int_{H=H^{\dagger}} d H f(H) e^{\eta \operatorname{Str}[H]} \int_{F=F^{\dagger}} d F e^{i F H} \mathcal{S} \operatorname{det}[\eta+i F]^{-N}
\end{aligned}
$$

where $d\left(\psi, \psi^{*}\right) \equiv \prod_{k}^{N} d \psi_{k} d \psi_{k}^{*}$ and $d \psi_{k} d \psi_{k}^{*}=\prod_{b}^{n_{b}} d_{\mathbb{C}}^{2} \psi_{k, b} \prod_{f}^{n_{f}} d \psi_{k, f} d \psi_{k, f}^{*}$. We denote by $\psi^{\dagger}=\psi^{* T}$, and $\psi \otimes \psi^{\dagger}$ is the external product and $\psi^{\dagger} \cdot \psi$ the scalar product in $\left(n_{b} \mid n_{f}\right)$. The measure $d H$ is the flat measure in the independent entries of the super-Hermitian matrix.

From now on let's focus on the second integral. This quantity reminds of a quantity already computed in [53], unfortunately we cannot use this result because it does not take care about the boundary terms arising from the diagonalisation of super-Hermitian matrices (Efetov-Wegner terms). In the following we will never change the $Z$-gradings of the integration manifold, and hence no boundary terms will arise 55 .

Writing $F$ and $H$ in terms of blocks, $d F=d F_{1} d F_{2} d \Phi d \Phi^{\dagger}$

$$
F=\left(\begin{array}{cc}
F_{1} & \Phi^{\dagger} \\
\Phi & F_{2}
\end{array}\right), \quad H=\left(\begin{array}{cc}
H_{1} & \Theta^{\dagger} \\
\Theta & H_{2}
\end{array}\right)
$$

we obtain:

$$
\begin{aligned}
\mathcal{I} & \equiv \int_{F=F^{\dagger}} d F e^{i F H} \mathcal{S} \operatorname{det}[F-i \eta]^{-N} \\
& =\int d F_{1} d F_{2} d \Phi d \Phi^{\dagger}\left(\frac{\operatorname{Det}\left[F_{2}-i \eta-\Phi\left(F_{1}-i \eta\right)^{-1} \Phi^{\dagger}\right]}{\operatorname{Det}\left[F_{1}-i \eta\right]}\right)^{N} \operatorname{Exp}\left[i \operatorname{Tr}\left[F_{1} H_{1}+\Phi^{\dagger} \Theta\right]-i \operatorname{Tr}\left[H_{2} F_{2}+\Phi \Theta^{\dagger}\right]\right] .
\end{aligned}
$$

\footnotetext{
${ }^{6}$ This theorem ensures that a bounded linear functional of functions over a locally compact Hausdorff space may be computed like an integration of that function over that space using a proper measure [54.

${ }^{7}$ This work was already presented by one of the authors in "QCD in extreme conditions", Frascati 6-8 August 2007.
} 
As a first step we note that if we diagonalise the matrix $F_{1}$ and make an analytic continuation in the eigenvalues we see that the only poles in the expression above are the ones where at least one eigenvalue of $F_{1}$ is $i \eta$. Considering the integration on the eigenvalues as a complex contour integral and closing the integral in the upper semicircle (or lower one, depending on the signature of the matrix $H_{1} 8$ ) we have non vanishing contributions only if every path of the eigenvalues winds the pole in $i \eta$. We can consider this integral as a contour integral around in whenever $\theta\left(H_{1}\right)>0$, otherwise it's zero. Since there are no poles apart from the ones in in we can fix the contour integral as we prefer. We choose the modulus of the eigenvalues as equal to 1 , hence we have that the matrix $F_{1}-i \eta$ may be analytically continued obtaining a unitary matrix.

$$
\begin{aligned}
\mathcal{I}= & \theta\left(H_{1}\right) e^{-\eta \operatorname{Tr}\left[H_{1}\right]} \oint_{U\left(n_{b}\right)} d F_{1} \int d F_{2} d \Phi d \Phi^{\dagger}\left(\frac{\operatorname{Det}\left[F_{2}-i \eta-\Phi F_{1}^{-1} \Phi^{\dagger}\right]}{\operatorname{Det}\left[F_{1}\right]}\right)^{N} \\
& \times \operatorname{Exp}\left[i\left[F_{1} H_{1}+\Phi^{\dagger} \Theta\right]-i\left[H_{2} F_{2}+\Phi \Theta^{\dagger}\right]\right] .
\end{aligned}
$$

The notation $\oint_{U\left(n_{b}\right)} d F_{1}$ stands for an integral over the manifold $U\left(n_{b}\right)$ considering as a measure the analytic continuation of the flat measure of Hermitian matrices. It is related to the Haar measure $d \mu_{U}$ integration by:

$$
\oint_{U\left(n_{b}\right)} d F_{1}=\int_{U\left(n_{b}\right)} d \mu_{U}\left(F_{1}\right) \operatorname{Det}\left[F_{1}\right]^{n_{b}} .
$$

The relation between these two integrals is the same as between the two integrals below describing a circuitation around zero, with $z=e^{i \theta}$ :

$$
\int_{0}^{2 \pi} d \theta=\oint_{|z|=1} d z \frac{1}{z} \neq \oint_{|z|=1} d z .
$$

Eq. (60) can be derived diagonalising the matrix $F_{1}=U \cdot \mathrm{f} \cdot U^{\dagger}$ where $\mathrm{f}_{j}=e^{i \theta_{j}}$ :

$$
\begin{aligned}
\oint_{U\left(n_{b}\right)} d F_{1} & \equiv \prod_{i} \oint d \mathrm{f}_{i} \Delta^{2}(\{\mathrm{f}\}) \int d U \\
& =\prod_{i} \oint \frac{d \mathrm{f}_{i}}{\mathrm{f}_{i}} \operatorname{Det}[\mathrm{f}] \Delta(\{\mathrm{f}\}) \Delta\left(\left\{\mathrm{f}^{-1}\right\}\right) \operatorname{Det}[\mathrm{f}]^{n_{b}-1} \int d U \\
& =\prod_{i} \int_{0}^{2 \pi} d \theta_{i}\left|\Delta\left(\left\{e^{i \theta}\right\}\right)\right|^{2} \operatorname{Det}[\mathrm{f}]^{n_{b}} \int d U \\
& \equiv \int_{U\left(n_{b}\right)} d \mu_{U}\left(F_{1}\right) \operatorname{Det}\left[F_{1}\right]^{n_{b}}
\end{aligned}
$$

We consider now that

$$
\begin{aligned}
\int_{A=A^{\dagger}} d A \operatorname{Det}[A]^{N} e^{-i \operatorname{Tr}\left[A H_{2}\right]} & =\int_{A=A^{\dagger}} d A \operatorname{Det}[A-i \eta]^{N} e^{-i \operatorname{Tr}\left[(A-i \eta) H_{2}\right]} \\
& =\int_{A=A^{\dagger}} d A \operatorname{Det}\left[A-i \eta-\Phi^{\dagger} F_{1}^{-1} \Phi\right]^{N} e^{-i \operatorname{Tr}\left[\left(A-i \eta-\Phi F_{1}^{-1} \Phi^{\dagger}\right) H_{2}\right]}
\end{aligned}
$$

The first equality comes from the analyticity of the integrand in the diagonal entries of the matrix and the second comes from the analogue of the contour invariance in superanalysis [56, applied to the real and imaginary parts of the Hermitian matrix entries. Applying this equivalence we obtain

$$
\begin{aligned}
\mathcal{I}= & \theta\left(H_{1}\right) e^{\eta \operatorname{Tr}\left[H_{2}\right]-\eta \operatorname{Tr}\left[H_{1}\right]} \oint_{U\left(n_{b}\right)} d F_{1} \int d F_{2} d \Phi d \Phi^{\dagger}\left(\frac{\operatorname{Det}\left[F_{2}\right]}{\operatorname{Det}\left[F_{1}\right]}\right)^{N} \\
& \times \operatorname{Exp}\left[i \operatorname{Tr}\left[F_{1} H_{1}+\Phi^{\dagger} \Theta\right]-i \operatorname{Tr}\left[H_{2} F_{2}+\Phi \Theta^{\dagger}\right]-i \operatorname{Tr}\left[H_{2} \Phi F_{1}^{-1} \Phi^{\dagger}\right]\right] .
\end{aligned}
$$

\footnotetext{
${ }^{8}$ The matrices $H_{1}$ and $H_{2}$ are complex number Hermitian matrices.
} 
The subsequent step is to perform the $\Phi, \Phi^{\dagger}$ integration, using

$$
\begin{aligned}
\operatorname{Tr}\left[\Phi^{\dagger} \Theta\right]-\operatorname{Tr}\left[\Phi \Theta^{\dagger}+H_{2} \Phi F_{1}^{-1} \Phi^{\dagger}\right] & =-\operatorname{Tr}\left[\Theta \Phi^{\dagger}+\Phi \Theta^{\dagger}+H_{2} \Phi F_{1}^{-1} \Phi^{\dagger}\right] \\
& =-\operatorname{Tr}\left[\Theta F_{1} F_{1}^{-1} \Phi^{\dagger}+H_{2} \Phi \Theta^{\dagger} H_{2}^{-1}+H_{2} \Phi F_{1}^{-1} \Phi^{\dagger}\right] \\
& =-\operatorname{Tr}\left[\left(\Theta F_{1}+H_{2} \Phi\right) \cdot\left(F_{1}^{-1} \Phi^{\dagger}+\Theta^{\dagger} H_{2}^{-1}\right)-\Theta F_{1} \Theta^{\dagger} H_{2}^{-1}\right] .
\end{aligned}
$$

We can transform the fermionic variables of integration $\Phi \rightarrow \tilde{\Phi} \equiv H_{2} \Phi+\Theta F_{1}$ and $\Phi^{\dagger} \rightarrow \tilde{\Phi}^{\dagger} \equiv F_{1}^{-1} \Phi^{\dagger}+$ $\Theta^{\dagger} H_{2}^{-1}$ and thus perform the Gaussian integration,

$$
\int d \Phi d \Phi^{\dagger} \operatorname{Exp}\left[-i \operatorname{Tr}\left[H_{2} \Phi F_{1}^{-1} \Phi^{\dagger}\right]+i \operatorname{Tr}\left[\Theta F_{1} \Theta^{\dagger} H_{2}^{-1}\right]\right]=\frac{\operatorname{Det}\left[H_{2}\right]^{n_{b}}}{\operatorname{Det}\left[F_{1}\right]^{n_{f}}} e^{i \operatorname{Tr}\left[\Theta F_{1} \Theta^{\dagger} H_{2}^{-1}\right]} \int d \tilde{\Phi} d \tilde{\Phi}^{\dagger} e^{-i \operatorname{Tr}\left[\tilde{\Phi} \tilde{\Phi}^{\dagger}\right]}
$$

where the last integration is just a constant. Getting back to eq. (64) we have:

$$
\begin{aligned}
\mathcal{I} & \propto \theta\left(H_{1}\right) \operatorname{Det}\left[H_{2}\right]^{n_{b}} e^{-\eta \operatorname{Str}[H]} \oint_{U\left(n_{b}\right)} d F_{1} \int d F_{2} \frac{\operatorname{Det}\left[F_{2}\right]^{N}}{\operatorname{Det}\left[F_{1}\right]^{N+n_{f}}} e^{i \operatorname{Tr}\left[F_{1} H_{1}\right]-i \operatorname{Tr}\left[H_{2} F_{2}\right]+i \operatorname{Tr}\left[\Theta F_{1} \Theta^{\dagger} H_{2}^{-1}\right]} \\
& =\theta\left(H_{1}\right) \operatorname{Det}\left[H_{2}\right]^{n_{b}} e^{-\eta \operatorname{Str}[H]} \int_{F_{2}=F_{2}^{\dagger}} d F_{2} \operatorname{Det}\left[F_{2}\right]^{N} e^{-i \operatorname{Tr}\left[H_{2} F_{2}\right]} \oint_{U\left(n_{b}\right)} d F_{1} \frac{1}{\operatorname{Det}\left[F_{1}\right]^{N+n_{f}}} e^{i\left[F_{1}\left(H_{1}-\Theta^{\dagger} H_{2}^{-1} \Theta\right)\right] .}
\end{aligned}
$$

Let us consider again the whole eq. (56). We can now perform the integration in $F_{1}$ and $F_{2}$, using the integrals in appendix B

$$
\begin{aligned}
\int d\left(\psi, \psi^{*}\right) f\left(\sum_{k} \psi_{k} \otimes \psi_{k}^{\dagger}\right) \propto & \int_{H_{1}=H_{1}^{\dagger}} d H_{1} \theta\left(H_{1}\right) \int_{H_{2}=H_{2}^{\dagger}} d H_{2} \operatorname{Det}\left[H_{2}\right]^{n_{b}} \int d \Theta d \Theta^{\dagger} f(H) \\
& \times \int_{F_{2}=F_{2}^{\dagger}} d F_{2} \operatorname{Det}\left[F_{2}\right]^{N} e^{-i \operatorname{Tr}\left[H_{2} F_{2}\right]} \oint_{U\left(n_{b}\right)} d F_{1} \frac{1}{\mathcal{D} \operatorname{Det}\left[F_{1}\right]^{N+n_{f}}} e^{i\left[F_{1}\left(H_{1}-\Theta^{\dagger} H_{2}^{-1} \Theta\right)\right]} \\
\propto & \int_{H_{1}=H_{1}^{\dagger}} d H_{1} \theta\left(H_{1}\right) \int_{H_{2}=H_{2}^{\dagger}} d H_{2} \operatorname{Det}\left[H_{2}\right]^{n_{b}} \int d \Theta d \Theta^{\dagger} f(H) \\
& \times \int_{F_{2}=F_{2}^{\dagger}} d F_{2} \operatorname{Det}\left[F_{2}\right]^{N} e^{-i T r\left[H_{2} F_{2}\right]} \operatorname{\mathcal {Det}}\left[H_{1}-\Theta^{\dagger} H_{2}^{-1} \Theta\right]^{N+n_{f}-n_{b}} \\
\propto & \int_{H_{1}=H_{1}^{\dagger}} d H_{1} \theta\left(H_{1}\right) \oint_{U\left(n_{f}\right)} d H_{2} \int d \Theta d \Theta^{\dagger} \frac{\operatorname{Det}\left[H_{1}-\Theta^{\dagger} H_{2}^{-1} \Theta\right]^{N+n_{f}-n_{b}}}{\operatorname{Det}\left[H_{2}\right]^{N+n_{f}-n_{b}} f(H)} \\
= & \int_{H_{1}=H_{1}^{\dagger}} d H_{1} \theta\left(H_{1}\right) \oint_{U\left(n_{f}\right)} d H_{2} \int d \Theta d \Theta^{\dagger} \mathcal{S} d e t\left[\begin{array}{cc}
H_{1} & \Theta^{\dagger} \\
\Theta & H_{2}
\end{array}\right]^{N+n_{f}-n_{b}} f(H) .(68)
\end{aligned}
$$

The last step missing is to find a relation between the measure used in the equation above and the Haar measure. This relation is:

$$
\int_{H_{1}=H_{1}^{\dagger}} d H_{1} \theta\left(H_{1}\right) \oint_{U\left(n_{f}\right)} d H_{2} \int d \Theta d \Theta^{\dagger} \mathcal{S} d e t\left[\begin{array}{cc}
H_{1} & \Theta^{\dagger} \\
\Theta & H_{2}
\end{array}\right]^{n_{f}-n_{b}}=\int_{\hat{G} l\left(n_{b} \mid n_{f}\right)} d \mu_{H}\left(\begin{array}{cc}
H_{1} & \Theta^{\dagger} \\
\Theta & H_{2}
\end{array}\right) .
$$

It is proved in the following. The manifolds we are integrating are the same, in fact the $H_{1}=H_{1}^{\dagger}>0$ is equivalent to the boson-boson [40] base manifold of $\hat{G} l\left(n_{b} \mid n_{f}\right)$ that is $G l\left(n_{b}\right) / U\left(n_{b}\right)$ [42, 29]. The analytic continuation of the eigenvalues of $H_{2}=H_{2}^{\dagger}$ making a circuitation around zero is equivalent to the fermion-fermion part $U\left(n_{f}\right)$ 42.

We have now to consider the integration measures in eq. (69). The point is that the measure on the l.h.s. is the Haar measure of super-Hermitian matrices (a group closed under addition) computed on the analytically continued manifold. It is induced by the flat metric:

$$
\operatorname{Str}[d U \cdot d U],
$$


but the Haar measure on the r.h.s. is the analytical continuation of the Haar measure on super-Unitary matrices [42, 40, 29] (a group closed under multiplication), induced by the metric [29]:

$$
\operatorname{Str}\left[d U \cdot d U^{-1}\right]=\operatorname{Str}\left[U^{-1} d U \cdot U^{-1} d U\right] .
$$

In order to find a relation between these two matrices one has to compute the Berezinean of the transformation [56, 42]:

$$
\mathfrak{B}=\operatorname{Sdet}_{i j, m n}\left[\frac{\left(U^{-1} d U\right)_{i j}}{d U_{m n}}\right] .
$$

Since this is not a change of variables (the differentials $d U$ are the same) and hence no Z-gradings are changed, no boundary (Efetov-Wegner) terms arise in the superintegration. We write this Berezinean matrix in the block structure (Latin indices stay for bosons and Greek for fermions):

$$
\begin{aligned}
\frac{U^{-1} d U}{d U} & =\left(\begin{array}{cccc}
b b / b b & b b / f f & b b / f b & b b / b f \\
f f / b b & f f / f f & f f / f b & f f / b f \\
f b / b b & f b / f f & f b / f b & f b / b f \\
b f / b b & b f / f f & b f / f b & b f / b f
\end{array}\right) \\
& =\left(\begin{array}{cccc}
U_{a b}^{-1} \otimes \mathbf{1}_{n_{b}} & 0 & U_{a \beta}^{-1} \otimes \mathbf{1}_{n_{b}} & 0 \\
0 & U_{\alpha \beta}^{-1} \otimes \mathbf{1}_{n_{f}} & 0 & U_{\alpha b}^{-1} \otimes \mathbf{1}_{n_{f}} \\
U_{\alpha b}^{-1} \otimes \mathbf{1}_{n_{b}} & 0 & U_{\alpha \beta}^{-1} \otimes \mathbf{1}_{n_{b}} & 0 \\
0 & U_{a \beta}^{-1} \otimes \mathbf{1}_{n_{f}} & 0 & U_{a b}^{-1} \otimes \mathbf{1}_{n_{f}}
\end{array}\right) .
\end{aligned}
$$

Computing the superdeterminant of the matrix above we obtain

$$
\mathfrak{B}=\mathcal{S}_{\operatorname{det}} t_{i j, m n}\left[\frac{\left(U^{-1} d U\right)_{i j}}{d U_{m n}}\right]=\mathcal{S} \operatorname{det}[U]^{n_{f}-n_{b}} .
$$

As a consequence we have:

$$
d \mu_{H}\left(\begin{array}{cc}
H_{1} & \Theta^{\dagger} \\
\Theta & H_{2}
\end{array}\right)=d H_{1} d H_{2} d \Theta d \Theta^{\dagger} \mathcal{S} \operatorname{det}\left[\begin{array}{cc}
H_{1} & \Theta^{\dagger} \\
\Theta & H_{2}
\end{array}\right]^{n_{f}-n_{b}},
$$

that is eq. (69). Together with eq. (68) we obtain the proof of our theorem eq. (55).

\section{Conclusions}

In the present paper we prove that $\chi \mathrm{PT}$ in the $\varepsilon$-regime and chRMT have the very same spectral properties in the microscopic (weak non-Hermiticity) limit. The equivalence holds for zero, imaginary and real chemical potentials. This result is achieved by proving the equivalence (up to an irrelevant overall constant) of the partition function of the partially quenched chRMT, with $n_{b}$ bosonic and $n_{f}$ fermionic quarks, to an integral defined over the maximal Riemannian subspace of the linear group of $\left(n_{b} \mid n_{f}\right)$ supermatrices for any finite $N$. Performing the $N \rightarrow \infty$ limit of this integral one obtains the partially quenched $\epsilon \chi \mathrm{PT}$. The one obtained for real chemical potential was derived here for the first time and agrees with the previously known purely fermionic and purely bosonic cases. An essential ingredient of this computation is a superbosonisation theorem connecting integrals of external products of supervectors to integrals over $\hat{G} l\left(n_{b} \mid n_{f}\right)$. An explicit parametrisation of integrals over $\hat{G} l\left(n_{b} \mid n_{f}\right)$ is provided as well.

The equivalence we have established by matching the generating functionals of density correlations holds also in cases where the densities are not yet known explicitly. Only for at most two different chemical potentials all densities have been derived so far. Our results also shed some light on universality in chRMT with chemical potential by matching the Gaussian one- and two-matrix model of Stephanov and Osborn, respectively. This gives hope to establish a further reaching universality beyond Gaussian RMT.

\section{Acknowledgements:}

We would like to thank P. H. Damgaard, A. Patella, K. Splittorff, B. Svetitsky and J. J. Verbaarschot for useful discussions, M. Zirnbauer for communications and sharing an unpublished paper, and the CPT Luminy for hospitality, where part of this work was being done. This work was supported by EU network ENRAGE MRTN-CT-2004-005616 (G.A.), EPSRC grant EP/D031613/1 and Brunel University BRIEF award no. 707. 


\section{A Defining $\delta$-functions on super-Hermitian matrices}

The question is whether or not one can define a $\delta$-distribution such that

$$
f\left(\sum_{k} \psi_{k} \otimes \psi_{k}^{\dagger}\right)=\int d M f(M) \delta\left(M-\sum_{k} \psi_{k} \otimes \psi_{k}^{\dagger}\right)
$$

In order to clarify the idea we take the same simple but nontrivial example as in [11, that is $k=1$, $\left(n_{b} \mid n_{f}\right)=(0 \mid 2)$. The external product is a $2 \times 2$ matrix, it's terms are only nilpotent commuting numbers $\psi_{i}$ and $\psi_{j}^{*}$ for $i, j=1,2$. We can consider this matrix as belonging to a superanalytic continuation of a manifold of real dimension 4 (at least). We could take as a such manifold $U(2), G l(2) / U(2)$, Hermitian matrices or real matrices. Despite in the rest of the work we have used Hermitian matrices, for this simple example we will use the one with the simplest notation, real matrices:

$$
a \equiv\left(\begin{array}{ll}
a_{11} & a_{12} \\
a_{21} & a_{22}
\end{array}\right) \equiv \psi \otimes \psi^{\dagger}=\left(\begin{array}{ll}
\psi_{1} \psi_{1}^{*} & \psi_{1} \psi_{2}^{*} \\
\psi_{2} \psi_{1}^{*} & \psi_{2} \psi_{2}^{*}
\end{array}\right)
$$

Once considering the external product of vectors like an element of a commuting number manifold, the well definiteness of the $\delta$-distribution is inherited from the one of the base manifold by superanalyticity in the real parameters of the manifold. In formulas:

$$
f(a) \equiv f\left(a_{11}, a_{12}, a_{21}, a_{22}\right)=\int d m \delta(m-a) f\left(m_{11}, m_{12}, m_{21}, m_{22}\right)
$$

where the integration manifold is the base manifold (real matrices) $\int d m \equiv \int_{-\infty}^{\infty} \prod_{i, j=1}^{2} d m_{i j}$, and the $\delta$ distribution is the one of the base manifold too, $\delta(m-a) \equiv \prod_{i, j=1}^{2} \delta\left(m_{i j}-a_{i j}\right)$.

The last thing to be verified is that the superanalytic continuation of the $\delta$-distribution is properly defined; this was already shown in [56]. Given a quantity $x=x_{B}+x_{S}$, where the first part is the body of the number, and the second is the nilpotent part. Given a function with a sufficient number of derivatives in $x_{B}$ such that $f\left(x_{B}+x_{S}\right)=\sum_{n=0}^{\infty} f^{(n)}\left(x_{B}\right) \frac{x_{S}^{n}}{n !}$ is properly defined, we have:

$$
\begin{aligned}
\int_{-\infty}^{+\infty} d y \delta\left(y-x_{B}-x_{S}\right) f(y) & \equiv \int_{-\infty}^{+\infty} d y \sum_{n=0}^{\infty} \delta^{(n)}\left(y-x_{B}\right) \frac{\left(-x_{S}\right)^{n}}{n !} f(y) \\
& =\sum_{n=0}^{\infty} \frac{\left(x_{S}\right)^{n}}{n !} \int_{-\infty}^{+\infty} d y \delta\left(y-x_{B}\right) f^{(n)}\left(x_{B}\right)=f\left(x_{B}+x_{S}\right)
\end{aligned}
$$

Completing the discussion on the example above we can write explicitly $\delta(m-a)$ :

$$
\delta(m-a)=\delta(m)-\sum_{i, j=1}^{2} \psi_{i} \psi_{j}^{*} \partial_{m_{i j}} \delta(m)+\psi_{1} \psi_{1}^{*} \psi_{2} \psi_{2}^{*}\left(\partial_{m_{11}} \partial_{m_{22}}+\partial_{m_{12}} \partial_{m_{21}}\right) \delta(m)
$$

where $m$ is a real number $2 \times 2$ matrix.

The generalisation for what is done above to eq. (176) is straightforward: one considers the external product as an element belonging to the superanalytic continuation of a manifold. The manifold we choose is the one of super-Hermitian matrices: the boson-boson and the fermion-fermion block are Hermitian matrices, and the $\delta$ is defined as the superanalytic continuation of the product of $\delta$ 's over the real and imaginary parts of the independent elements of the matrices; boson-fermion and fermion-boson blocks are made of independent Grassmann integration variables and $\delta$ may be represented as:

$$
\delta(\theta-\tilde{\theta}) \propto(\theta-\tilde{\theta}) \propto \int d \xi e^{i \xi(\theta-\tilde{\theta})}
$$


As an exponential representation of $\delta$ is allowed both for commuting and anticommuting variables, such a representation of $\delta$-function on super-Hermitian matrices is allowed too:

$$
\begin{aligned}
f(a) & =\int_{M=M^{\dagger}} d M f(M) \delta(M-a) \\
& \propto \int_{M=M^{\dagger}} d M f(M) \int_{F=F^{\dagger}} d F e^{i \operatorname{Str}[F(M-a)]}
\end{aligned}
$$

where $d M$ and $d F$ stand for the flat measure over the independent entries of super-Hermitian matrices:

$$
d M \equiv \prod_{i=1}^{n_{b}} \prod_{\alpha=1}^{n_{f}} d M_{i, \alpha} d M_{\alpha, i} \prod_{i=1}^{n_{b}} d M_{i, i} \prod_{i>j} d \mathbb{R} e M_{i, j} d \amalg m M_{i, j} \prod_{\alpha=1}^{n_{f}} d M_{\alpha, \alpha} \prod_{\alpha, \beta} d \mathbb{R} e M_{\alpha, \beta} d \mathbb{I} m M_{\alpha, \beta}
$$

\section{B The integrals in eq. (67)}

\section{B.1 Boson-boson block}

Let's start from

$$
\begin{aligned}
& \oint_{U\left(n_{b}\right)} d F_{1} \frac{1}{\mathcal{D e t}\left[F_{1}\right]^{N+n_{f}}} \operatorname{Exp}\left[i \operatorname{Tr}\left[F_{1} \tilde{H}\right]\right]= \\
& =\int_{U\left(n_{b}\right)} d \mu_{U}\left(F_{1}\right) \operatorname{Det}\left[F_{1}\right]^{-N-n_{f}+n_{b}} \operatorname{Exp}\left[i \operatorname{Tr}\left[F_{1} \tilde{H}\right]\right] \\
& =\operatorname{Det}[\tilde{H}]^{N+n_{f}-n_{b}} \int_{U\left(n_{b}\right)} d \mu_{U}\left(F_{1}\right) \operatorname{Det}\left[F_{1} \tilde{H}\right]^{-N-n_{f}+n_{b}} \operatorname{Exp}\left[i \operatorname{Tr}\left[F_{1} \tilde{H}\right]\right]
\end{aligned}
$$

where for simplicity of notation we use $\tilde{H}=H_{1}-\Theta H_{2}^{-1} \Theta^{\dagger}$. The integral in the last line may be performed using the character expansion. This is a particular case of a more general integral computed in [57]; the result is:

$$
\sum_{r} \frac{\alpha_{r}^{(0)}}{d_{r}} \alpha_{r}^{\left(N+n_{f}-n_{b}\right)} \chi_{r}(0)
$$

where the sum is over the irreducible representations of $G l\left(n_{b}\right)$. The quantity $\chi_{r}(0)$ is zero for all the representation apart from the trivial one. The result is just a constant 9 .

$$
\oint_{U\left(n_{b}\right)} d F_{1} \frac{1}{\mathcal{D} e t\left[F_{1}\right]^{N+n_{f}}} e^{i \operatorname{Tr}\left[F_{1} \tilde{H}\right]} \propto \mathcal{D} e t[\tilde{H}]^{N+n_{f}-n_{b}} .
$$

This result, together with the argument of analytic continuation of Hermitian matrices, gives an alternative way for computing the Ingham-Siegel integral as was done in [27.

\section{B.2 Fermion-fermion block}

The fermion-fermion block integral has already been computed by Guhr [53]. The result is:

$$
\int_{F_{2}=F_{2}^{\dagger}} d F_{2} \operatorname{Det}\left[F_{2}\right]^{N} \operatorname{Exp}\left[-i \operatorname{Tr}\left[F_{2} H_{2}\right]\right] \propto \prod_{j} \delta^{\left(N+n_{f}-1\right)}\left(h_{j}\right) .
$$

Here we don't have any problem with possible Efetov-Wegner terms arising from these diagonalisation: both $\mathrm{F}_{2}$ and $\mathrm{H}_{2}$ are complex Hermitian matrices and no Z-grading will be changed during diagonalisation [55].

\footnotetext{
${ }^{9}$ In order to compute the exact value one has just to substitute the relation $\alpha_{r}^{(\nu)}=\operatorname{det}_{i j}\left[\frac{1}{n_{j}-\nu+i-j}\right]$, where $r=\left(n_{1}, \ldots, n_{b}\right)$ are the labels of the representation.
} 
If we integrate this functional with a given function analytic in the matrix entries, using the Cauchy integral formula we obtain:

$$
\begin{aligned}
\int d \mu_{U}(U) \prod_{j} \int d h_{j} \Delta(\{h\})^{2} g\left(U h U^{\dagger}\right) \prod_{j} \delta^{\left(N+n_{f}-1\right)}\left(h_{j}\right) & \propto \int d \mu_{U}(U) \oint \prod_{j} d h_{j} \frac{1}{h_{j}^{N+n_{f}}} \Delta(\{h\})^{2} g\left(U h U^{\dagger}\right) \\
& =\oint_{U\left(N_{f}\right)} d H_{2} \frac{1}{\operatorname{Det}\left[H_{2}\right]^{N+n_{f}}} g\left(H_{2}\right) .
\end{aligned}
$$

\section{References}

[1] E. V. Shuryak and J. J. M. Verbaarschot, Nucl. Phys. A 560 (1993) 306 arXiv:hep-th/9212088.

[2] J.J.M. Verbaarschot and T. Wettig, Ann. Rev. Nucl. Part. Sci. 50 (2000) 343 arXiv:hep-ph/0003017.

[3] G. Akemann, Int. J. Mod. Phys. A 22 (2007) 1077 arXiv:hep-th/0701175.

[4] J. J. M. Verbaarschot, Phys. Rev. Lett. 72 (1994) 2531 arXiv:hep-th/9401059.

[5] A. Smilga and J. J. M. Verbaarschot, Phys. Rev. D 51 (1995) 829 arXiv:hep-th/9404031.

[6] D. Toublan and J. J. M. Verbaarschot, Nucl. Phys. B 560 (1999) 259 arXiv:hep-th/9904199.

[7] J. C. Osborn, Phys. Rev. Lett. 93 (2004) 222001 arXiv:hep-th/0403131.

[8] G. Akemann, P. H. Damgaard, J. C. Osborn and K. Splittorff, Nucl. Phys. B 766 (2007) 34 arXiv:hep-th/0609059.

[9] M. A. Stephanov, Phys. Rev. Lett. 76 (1996) 4472 arXiv:hep-lat/9604003].

[10] P. Littelmann, H.-J. Sommers and M. R. Zirnbauer, arXiv:0707.2929.

[11] J.E. Bunder, K.B. Efetov, V.E. Kravtsov, O.M. Yevtushenko and M.R. Zirnbauer, arXiv:0707.2932

[12] T. Guhr, A. Müller-Groeling and H. A. Weidenmüller, Phys. Rept. 299 (1998) 189 arXiv:cond-mat/9707301].

[13] M.L. Mehta, Random Matrices, Academic Press, Third Edition, London 2004.

[14] K. Splittorff and J. J. M. Verbaarschot, Phys. Rev. Lett. $90 \quad$ (2003) 041601 arXiv:cond-mat/0209594.

[15] Y. V. Fyodorov and G. Akemann, JETP Lett. 77 (2003) 438 [Pisma Zh. Eksp. Teor. Fiz. 77 (2003) 513] arXiv:cond-mat/0210647.

[16] G. Akemann, J. C. Osborn, K. Splittorff and J. J. M. Verbaarschot, Nucl. Phys. B 712 (2005) 287 arXiv:hep-th/0411030.

[17] K. Splittorff and J. J. M. Verbaarschot, Nucl. Phys. B 757 (2006) 259 arXiv:hep-th/0605143.

[18] G. Akemann, P. H. Damgaard, U. Magnea and S. Nishigaki, Nucl. Phys. B 487 (1997) 721 arXiv:hep-th/9609174.

[19] P. H. Damgaard and S. M. Nishigaki, Nucl. Phys. B 518 (1998) 495 arXiv:hep-th/9711023.

[20] T. Wilke, T. Guhr and T. Wettig, Phys. Rev. D 57 (1998) 6486 arXiv:hep-th/9711057.

[21] S. M. Nishigaki, P. H. Damgaard and T. Wettig, Phys. Rev. D 58 (1998) 087704 arXiv:hep-th/9803007; P. H. Damgaard and S. M. Nishigaki, Phys. Rev. D 63 (2001) 045012 arXiv:hep-th/0006111. 
[22] G. Akemann, J. Bloch L. Shifrin and T. Wettig, PoS(Lattice2007)224, arXiv:0711.0629.

[23] G. Akemann and P. Damgaard, PoS(Lattice2007)166, arXiv:0709.0484.

[24] K. Splittorff and J. J. M. Verbaarschot, Nucl. Phys. B 683 (2004) 467 arXiv:hep-th/0310271].

[25] G. Akemann, Y.V. Fyodorov and G. Vernizzi, Nucl. Phys. B 694 (2004) 59 arXiv:hep-th/0404063.

[26] J. C. Osborn, PoS LAT2006 (2006) 142 arXiv:hep-lat/0610016.

[27] Y. V. Fyodorov, Nucl. Phys. B 621 (2002) 643 arXiv:math-ph/0106006.

[28] J. C. Osborn, D. Toublan and J. J. M. Verbaarschot, Nucl. Phys. B 540 (1999) 317 arXiv:hep-th/9806110.

[29] P. H. Damgaard, J. C. Osborn, D. Toublan and J. J. M. Verbaarschot, Nucl. Phys. B 547 (1999) 305 arXiv:hep-th/9811212.

[30] D. Toublan and J. J. M. Verbaarschot, Nucl. Phys. B 603 (2001) 343 arXiv:hep-th/0012144.

[31] P. H. Damgaard, U. M. Heller, K. Splittorff and B. Svetitsky, Phys. Rev. D 72 (2005) 091501 arXiv:hep-lat/0508029 ; P. H. Damgaard, U. M. Heller, K. Splittorff, B. Svetitsky and D. Toublan, Phys. Rev. D 73 (2006) 074023 arXiv:hep-lat/0602030; Phys. Rev. D 73 (2006) 105016 arXiv:hep-th/0604054.

[32] G. Akemann and P. H. Damgaard, Phys. Lett. B 583 (2004) 199 arXiv:hep-th/0311171.

[33] E. Kanzieper and V. Freilikher, Phil. Mag. B 77 (1998) 1161 arXiv:cond-mat/9704149]; A.B.J. Kuijlaars and M. Vanlessen, Commun. Math. Phys. 243 (2003) 163 arXiv:math-ph/0305044.

[34] R. J. Szabo, Nucl. Phys. B 598 (2001) 309 arXiv:hep-th/0009237.

[35] J. J. M. Verbaarschot, Lectures at the Les Houches Summer School on Applications of Random Matrices in Physics, 2004, arXiv:hep-th/0502029.

[36] N. I. Muskhelishvili, Singular Integral Equations Groningen : Noordhoff, 1953.

[37] D. Toublan and J. J. M. Verbaarschot, Int. J. Mod. Phys. B 15 (2001) 1404 arXiv:hep-th/0001110.

[38] Yan V. Fyodorov, H.-J. Sommers, JETP Lett. 63 (1996) 1026 arXiv:cond-mat/9507117.

[39] J. J. M. Verbaarschot, AIP Conf. Proc. 744 (2005) 277 arXiv:hep-th/0410211.

[40] D. Dalmazi and J. J. M. Verbaarschot, Nucl. Phys. B 592 (2001) 419 arXiv:hep-th/0005229.

[41] H. Leutwyler and A. Smilga, Phys. Rev. D 46 (1992) 5607.

[42] M. R. Zirnbauer, J. Math. Phys. 37 (1996) 4986 arXiv:math-ph/9808012.

[43] A. V. Andreev, B. D. Simons and N. Taniguchi, Nucl. Phys. B 432 (1994) 487.

[44] Y.V. Fyodorov, B.A. Khoruzhenko and H.-J. Sommers, Phys. Lett. A 226 (1997) 46 arXiv:cond-mat/9606173; Phys. Rev. Lett. 79 (1997) 557 arXiv:cond-mat/9703152.

[45] J. Feinberg and A. Zee, Nucl. Phys. B 504 (1997) 579 arXiv:cond-mat/9703087.

[46] R. A. Janik, M. A. Nowak, G. Papp and I. Zahed, Nucl. Phys. B 501 (1997) 603 arXiv:cond-mat/9612240].

[47] G. Akemann and A. Pottier, J. Phys. A 37 (2004) L453 arXiv:math-ph/0404068.

[48] N. Kawamoto and J. Smit, Nucl. Phys. B 192 (1981) 100. 
[49] G. Hackenbroich and H. A. Weidenmüller, Phys. Rev. Lett. $\mathbf{7 4}$ (1995) 4118 arXiv:cond-math/9412088.

[50] N. Lehmann, D. Saher, V. V. Sokolov and H. -J. Sommers, Nucl. Phys. A 582 (1995), 223.

[51] F. Berruto, R. C. Brower and B. Svetitsky, Phys. Rev. D 64 (2001) 114504 arXiv:hep-lat/0105016.

[52] K. B. Efetov, G. Schwiete, K. Takahashi, Phys. Rev. Lett. 92, (2004), 026807. arXiv:cond-mat/0307504.

[53] T. Guhr, J. Phys. A 39 (2006) 13191 arXiv:math-ph/0606014.

[54] W. Rudin, Real and Complex Analysis McGraw-Hill 1970.

[55] M. J. Rothstein, Trans. Am. Math. Soc. 299 (1987) 387.

[56] B. DeWitt, Supermanifolds, Cambridge University Press 1984.

[57] B. Schlittgen and T. Wettig, J. Phys. A 36 (2003) 3195 arXiv:math-ph/0209030. 